Research Article

\title{
Linear Active Disturbance Rejection Control of a Two-Degrees-of-Freedom Manipulator
}

\author{
Dawei Liu, Qinhe Gao $(\mathbb{D}$, Zhixiang Chen $(\mathbb{D}$, and Zhihao Liu \\ College of Missile Engineering, Rocket Force University of Engineering, Xi'an 710025, China \\ Correspondence should be addressed to Qinhe Gao; gao202@189.cn
}

Received 12 January 2020; Accepted 27 February 2020; Published 6 May 2020

Academic Editor: Jean Jacques Loiseau

Copyright (c) 2020 Dawei Liu et al. This is an open access article distributed under the Creative Commons Attribution License, which permits unrestricted use, distribution, and reproduction in any medium, provided the original work is properly cited.

\begin{abstract}
This paper presents linear active disturbance rejection control (LADRC) for a two-degrees-of-freedom (2-DOF) manipulator system to achieve trajectory tracking. The system is widely used in engineering applications and exhibits the characteristics of high nonlinearity, strong coupling, and large uncertainty with two inputs and two outputs. First, the problem of dynamic coupling in the model of the 2-DOF manipulator is addressed by considering the dynamic coupling, model uncertainties, and external disturbances as total disturbances. Second, a linear extended state observer is designed to estimate the total disturbances, while a linear state error feedback control law is designed to compensate these disturbances. The main contribution is that the stability of the closed-loop system with two inputs and two outputs is analyzed, and the relationship between the performance of the closedloop system and the controller parameters is established. The joint simulation of SolidWorks and Matlab/Simulink is conducted. The simulation and experimental results clearly indicate the superiority of LADRC over the PID for trajectory tracking and dynamic performance.
\end{abstract}

\section{Introduction}

With the rapid development of automation, manipulators are widely used in industries for applications such as assembling, picking, painting, and welding. Therefore, the trajectory tracking control of a manipulator has been widely studied. One challenge in achieving an acceptable performance (such as small errors, good trajectory, and disturbance rejection) might arise from uncertainty, high nonlinearity, and dynamic coupling in robotic systems $[1,2]$.

Focusing on the trajectory tracking control problem of a manipulator system, various control strategies have been studied in related fields, such as PD control with feedforward compensation [3,4], adaptive control [5-7], fuzzy control $[8,9]$, neural network control [10-13], and sliding mode control [14]. Chen et al. [3] proposed a modified PD feedforward compensation control. By using feedforward compensation in the PD control structure, the control accuracy of the manipulator is effectively improved. Kuc and Han [5] combined adaptive control with PID control and proposed an adaptive PID learning controller that was based on feedforward input learning. Ouyang et al. [6] proposed an adaptive switching learning PD control (ASLPD), which was a combination of the feedback PD control law with a gain switching technique and the feedforward learning control law with the input torque profile based on interative learning. According to the ASL-PD method, the convergence rate of manipulator trajectory tracking is effectively increased. The control of an uncertain robotic manipulator with input saturation was studied by Tran and Sam [7]. They introduced a model reference adaptive control-like (MRAC-like) to deal with the problems of input saturation, unknown input scaling, and disturbances. Lin et al. [9] proposed a variable structure control method to compensate for the uncertain part of the robotic manipulator and achieved the elimination of variable structure chattering by introducing the fuzzy control method. In order to improve the tracking precision of the flexible-link manipulator, $\mathrm{Xu}$ [12] proposed the DOB- 
based composite neural control to deal with unknown dynamics and time-varying disturbances. Considering the influence of gravity change, Liu et al. [14] designed a sliding mode controller (SMC) for a space manipulator. To solve the influence of the uncertain part of the manipulator, several studies combined the neural network with the PD control, fuzzy control, adaptive control, and sliding mode control compensating for the uncertain part $[4,8,11,15]$. However, the structure of the controllers became complicated as a result of the increase in the number of neural networks.

The abovementioned control strategies have had a certain effect on solving the uncertainty and nonlinearity of the manipulator; however, the problem of dynamic coupling has not been solved effectively. There are certain shortcomings in each control strategy influencing the performance of the systems [16]:

(1) A contradiction between system rapidity and overshoot in PID control, which has a limited effect on nonlinear characteristics and time-varying disturbances

(2) The adaptive control method requires the control object model to be sufficiently accurate and the tuning of the controller parameters is difficult

(3) The structure of fuzzy control and neural network control is complicated and the controller parameters are hard to tune

The combination of the control algorithms can make up for the disadvantages of each other to a certain extent; however, the problem of the complex structure of controllers cannot be solved.

Active disturbance rejection control (ADRC) was proposed by Han in 1995. It is almost independent of an accurate and detailed dynamic model of the plant and has strong robustness to nonlinear, uncertainties, and external disturbances [17]. The most prominent feature of ADRC is that all the uncertainties of the plant and external disturbances are considered as total disturbances, which are estimated by extended state observer (ESO) and compensated by linear state error feedback control law (NSEFCL). The traditional ADRC has many parameters, which makes the controller tuning complex. To simplify the tuning, Gao proposed Linear ADRC (LADRC) and used linear ESO (LESO) $[18,19]$. ADRC is widely applied because of its excellent performance of disturbance rejection and the convenience of engineering realization [20-24]. In recent years, remarkable progress has been made in the theoretical basis of ADRC. Zhao and Guo $[25,26]$ analyzed the stability of nonlinear active disturbance rejection control (NLADRC). Xue et al. [27-30] analyzed the performance of LADRC and provided theoretical references for an LADRC of a twodegrees-of-freedom (2-DOF) manipulator.

The rest of the contents are organized as follows. First, the mathematical model of the 2-DOF manipulator is established, which is based on the model decoupling. Section 3 plans the trajectory of a regular octagonal for the manipulator. The LADRC controller based on the decoupling system is presented. Boundedness of the estimation error and the closed-loop tracking error are then established. Furthermore, the estimation error and the closed-loop tracking error monotonously decrease with the two observers' and the two controllers' bandwidths, respectively. In addition, the joint simulation of SolidWorks and Matlab/Simulink is conducted. Furthermore, the simulation and the experiment demonstrate the better trajectory tracking and dynamic performance of LADRC than that of PID.

\section{Modeling and Trajectory Planning of a 2-DOF Manipulator System}

2.1. Modeling. The simplified model of the 2-DOF manipulator is depicted in Figure 1.

The dynamic model can be derived using the Euler-Lagrange dynamic equation, which comprises two coupled nonlinear second-order differential equations. Define $\theta=\left[\theta_{1}, \theta_{2}\right]^{T}$, and the model can be represented as

$$
\left\{\begin{array}{l}
M_{11} \ddot{\theta}_{1}+M_{12} \ddot{\theta}_{2}+N_{1}(\theta, \dot{\theta})+G_{1}(\theta)=u_{1}+d_{1}, \\
M_{21} \ddot{\theta}_{1}+M_{22} \ddot{\theta}_{2}+N_{2}(\theta, \dot{\theta})+G_{2}(\theta)=u_{2}+d_{2},
\end{array}\right.
$$

where

$$
\begin{aligned}
M_{11} & =a_{1}+a_{2} \cos \theta_{2}, \\
M_{12} & =M_{21}=a_{3}+\frac{1}{2} a_{2} \cos \theta_{2}, \\
M_{22} & =a_{3}, \\
N_{1}(\theta, \dot{\theta}) & =-a_{2} \sin \theta_{2}\left(\dot{\theta}_{1} \dot{\theta}_{2}+\frac{1}{2} \dot{\theta}_{2}^{2}\right), \\
N_{2}(\theta, \theta) & =\frac{1}{2} a_{2} \sin \theta_{2} \dot{\theta}_{1}^{2}, \\
G_{1}(\theta) & =a_{4} \sin \theta_{1}+a_{5} \sin \left(\theta_{1}+\theta_{2}\right), \\
G_{2}(\theta) & =a_{5} \sin \left(\theta_{1}+\theta_{2}\right), \\
a_{1} & =\frac{1}{3} m_{1} l_{1}^{2}+\frac{1}{3} m_{2} l_{2}^{2}+m_{2} l_{1}^{2}, \\
a_{2} & =m_{2} l_{2} l_{1}, \\
a_{3} & =\frac{1}{3} m_{2} l_{2}^{2}, \\
a_{4} & =\frac{1}{2} m_{1} l_{1} g+m_{2} l_{1} g, \\
a_{5} & =\frac{1}{2} m_{2} l_{2} g
\end{aligned}
$$




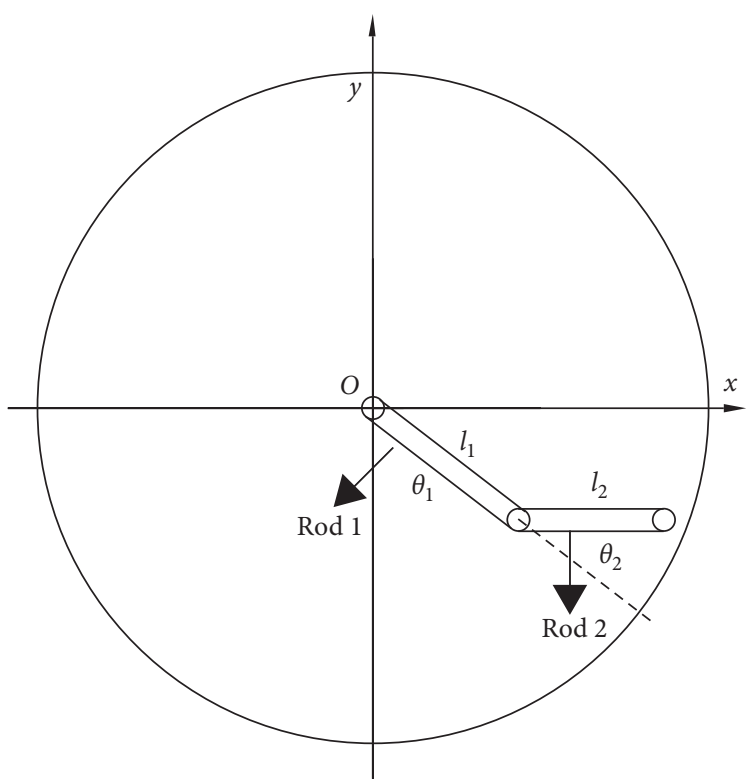

FIgURE 1: Simplified model of the 2-DOF manipulator.

where $l_{1}$ and $l_{2}$ represents the length of the 2 rods, $\theta_{1}$ and $\theta_{2}$ are the joint angles shown in Figure 1, $m_{1}$ and $m_{2}$ represent the mass of the 2 rods, $u_{1}$ and $u_{2}$ are the control laws of rod 1 and rod 2, respectively, and $d_{1}$ and $d_{2}$ represent the external disturbance of 2 rods, respectively.

2.2. Trajectory Planning. The trajectory planning and desired signal generation of the manipulator must be achieved before designing the LADRC, and they can be achieved as follows.

Assuming that the reachable area of the end effector is $\left\{(x, y) \in \mathbb{R}^{2} \mid x^{2}+y^{2} \leq\left(l_{1}+l_{2}\right)^{2}\right\}$, trajectory planning allows the end effector to run a desired trajectory in the area where the controlled objects are the motors that drive the two link rods to rotate.

Assuming that the end effector moves from the initial point $A=\left(x_{A}, y_{A}\right)=\left(0,-l_{1}-l_{2}\right)$ to another point $B=\left(x_{r}, y_{r}\right)$, the displacement in the $x$ and $y$ directions can be written as

$$
\begin{aligned}
& l_{x}=x_{r}-x_{A}=x_{r}, \\
& l_{y}=y_{r}-y_{A}=y_{r}+\left(l_{1}+l_{2}\right) .
\end{aligned}
$$

From point $\mathrm{A}$ to point $\mathrm{B}$, the motion in $x$ and $y$ directions is planned using the method of quintic polynomial [31]. Thus, the displacement, velocity, and acceleration in the $x$ and $y$ directions can be represented in the quintic form as

$$
\begin{aligned}
& l_{x}=a_{1}(1)+a_{1}(2) \cdot t+a_{1}(3) \cdot t^{2}+a_{1}(4) \cdot t^{3}+a_{1}(5) \cdot t^{4}+a_{1}(6) \cdot t^{5}, \\
& v_{x}=a_{1}(2)+2 \cdot a_{1}(3) \cdot t+3 \cdot a_{1}(4) \cdot t^{2}+4 \cdot a_{1}(5) \cdot t^{3}+5 \cdot a_{1}(6) \cdot t^{4}, \\
& a_{x}=2 \cdot a_{1}(3)+6 \cdot a_{1}(4) \cdot t+12 \cdot a_{1}(5) \cdot t^{2}+20 \cdot a_{1}(6) \cdot t^{3}, \\
& l_{y}=a_{2}(1)+a_{2}(2) \cdot t+a_{2}(3) \cdot t^{2}+a_{2}(4) \cdot t^{3}+a_{2}(5) \cdot t^{4}+a_{2}(6) \cdot t^{5}, \\
& v_{y}=a_{2}(2)+2 \cdot a_{2}(3) \cdot t+3 \cdot a_{2}(4) \cdot t^{2}+4 \cdot a_{2}(5) \cdot t^{3}+5 \cdot a_{2}(6) \cdot t^{4}, \\
& a_{y}=2 \cdot a_{2}(3)+6 \cdot a_{2}(4) \cdot t+12 \cdot a_{2}(5) \cdot t^{2}+20 \cdot a_{2}(6) \cdot t^{3},
\end{aligned}
$$


where $a_{1}=A^{-1} \cdot b_{1}, a_{2}=A^{-1} \cdot b_{2}$,

$$
\begin{aligned}
A & =\left[\begin{array}{cccccc}
1 & t_{o} & t_{o}^{2} & t_{o}^{3} & t_{o}^{4} & t_{o}^{5} \\
0 & 1 & 2 t_{o} & 3 t_{o}^{2} & 4 t_{o}^{3} & 5 t_{o}^{4} \\
0 & 0 & 2 & 6 t_{o} & 12 t_{o}^{2} & 20 t_{o}^{3} \\
1 & t_{f} & t_{f}^{2} & t_{f}^{3} & t_{f}^{4} & t_{f}^{5} \\
0 & 1 & 2 t_{f} & 3 t_{f}^{2} & 4 t_{f}^{3} & 5 t_{f}^{4} \\
0 & 0 & 2 & 6 t_{f} & 12 t_{f}^{2} & 20 t_{f}^{3}
\end{array}\right], \\
b_{1} & =\left[\begin{array}{llllll}
x_{A} & 0 & 0 & x_{r} & 0 & 0
\end{array}\right], \\
b_{2} & =\left[\begin{array}{llllll}
y_{A} & 0 & 0 & y_{r} & 0 & 0
\end{array}\right],
\end{aligned}
$$

where $t_{0}$ is the time at the initial point $\mathrm{A}, t_{f}$ is time to reach the point $\mathrm{B}, x_{A}$ and $y_{A}$ represent the positions of the end effector in $x$ and $y$ directions at the initial point, respectively, and $x_{r}$ and $y_{r}$ are the positions of the $x$ and the $y$ directions at the point of arrival.

From (3) and (4), the planned trajectory can be written as

$$
\begin{aligned}
& \qquad\left\{\begin{array}{l}
x_{r}(t)=x_{A}+l_{x}(t) \\
=a_{1}(1)+a_{1}(2) \cdot t+a_{1} \\
y_{r}(t)=y_{A}+l_{y}(t) \\
=a_{2}(1)+a_{2}(2) \cdot t+a_{2}
\end{array}\right. \\
& \text { and (6), we can obtain the desired } \\
& \text { 2-DOF manipulator, we have } \\
& x=l_{1} \sin \theta_{1}+l_{2} \sin \left(\theta_{1}+\theta_{2}\right), \\
& y=-l_{1} \cos \theta_{1}-l_{2} \cos \left(\theta_{1}+\theta_{2}\right) .
\end{aligned}
$$

and from (4) and (6), we can obtain the desired signal.

According to the structure and coordinate system definition of the 2-DOF manipulator, we have

From (7), the corresponding Jacobian matrix $J$ can be calculated as
$(4) \cdot t^{3}+a_{1}(5) \cdot t^{4}+a_{1}(6) \cdot t^{5}$

$(4) \cdot t^{3}+a_{2}(5) \cdot t^{4}+a_{2}(6) \cdot t^{5}-l_{1}-l_{2}$

$$
J=\left[\begin{array}{ll}
l_{1} \cos \theta_{1}+l_{2} \cos \left(\theta_{1}+\theta_{2}\right) & l_{2} \cos \left(\theta_{1}+\theta_{2}\right) \\
l_{1} \sin \theta_{1}+l_{2} \sin \left(\theta_{1}+\theta_{2}\right) & l_{2} \sin \left(\theta_{1}+\theta_{2}\right)
\end{array}\right],
$$

and let $v=[\dot{x} \dot{y}]^{T}, a=[\ddot{x} \ddot{y}]^{T}, \omega=\left[\dot{\theta}_{1} \dot{\theta}_{2}\right]^{T}$, and $\gamma=\left[\ddot{\theta}_{1} \ddot{\theta}_{2}\right]^{T}$; we have

$$
\begin{aligned}
& \nu=J \omega, \\
& \bar{a}=J \gamma,
\end{aligned}
$$

where

$$
\bar{a}=\left[\begin{array}{l}
\ddot{x}+\left(l_{1} \sin \theta_{1} \dot{\theta}_{1}+l_{2} \sin \left(\theta_{1}+\theta_{2}\right)\left(\dot{\theta}_{1}+\dot{\theta}_{2}\right)\right) \dot{\theta}_{1}+l_{2} \sin \left(\theta_{1}+\theta_{2}\right)\left(\dot{\theta}_{1}+\dot{\theta}_{2}\right) \dot{\theta}_{2} \\
\ddot{y}-\left(l_{1} \cos \theta_{1} \dot{\theta}_{1}+l_{2} \cos \left(\theta_{1}+\theta_{2}\right)\left(\dot{\theta}_{1}+\dot{\theta}_{2}\right)\right) \dot{\theta}_{1}-l_{2} \cos \left(\theta_{1}+\theta_{2}\right)\left(\dot{\theta}_{1}+\dot{\theta}_{2}\right) \dot{\theta}_{2}
\end{array}\right] .
$$

From (9), the desired velocity signal of the 2-DOF manipulator can be written as

$$
\begin{aligned}
& \omega_{r}=J^{-1} v_{r}, \\
& \gamma_{r}=J^{-1} \bar{a}_{r},
\end{aligned}
$$

and the desired angle signal can be written as

$$
\theta_{r}(t)=\int_{0}^{t} \omega_{r}(\tau) \mathrm{d} \tau
$$

Considering the case where $J$ is irreversible, assuming

$$
|J|=l_{1} l_{2} \sin \theta_{2}=0,
$$

we can obtain $\theta_{2}=2 k \pi, k \in \mathbb{N}$, when $J$ is irreversible. This situation must be avoided. Hence, when $\left|\theta_{2}-2 k \pi\right| \leq \delta \in \mathbb{R}^{+}$, letting $\dot{\theta}_{2}=c_{2} \in \mathbb{R}$, the system will avoid the irreversible region of matrix $J$. In trajectory planning, we should avoid reaching the irreversible region, which is the outer circle and the inner circle of the collinear point of the two link rods. The irreversible region can be written as

$$
\left\{(x, y) \in \mathbb{R}^{2} \mid x^{2}+y^{2}=\left(l_{1}+l_{2}\right)^{2}\right\} \cup\left\{(x, y) \in \mathbb{R}^{2} \mid x^{2}+y^{2}=\left(l_{1}-l_{2}\right)^{2}\right\} .
$$

To avoid any calculation error, (11) is modified as

$$
\omega_{r}=J^{-1}\left(v_{r}+\alpha\left(x_{r}-x\right)\right),
$$

where $\alpha$ is error gain.

The desired signal generation principle of the 2-DOF manipulator is depicted in Figure 2. The desired trajectory planned in this study is a regular octagonal.

\section{Design of LADRC}

3.1. Problem Description. Before designing an LADRC, we describe its mathematical model in (1). Letting $N G_{1}=N_{1}+$ $G_{1}$ and $N G_{2}=N_{2}+G_{2}$, (1) becomes 


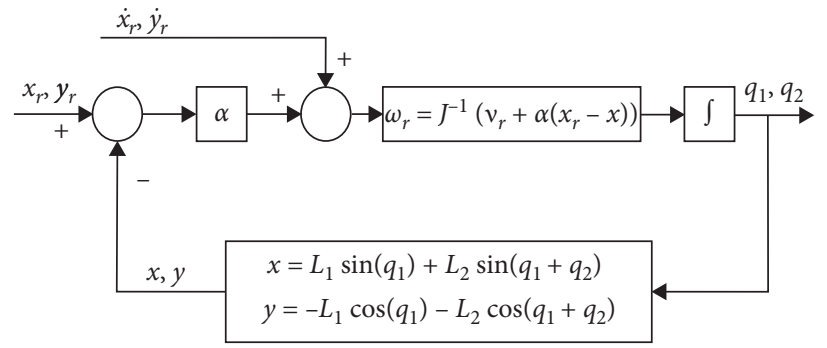

Figure 2: Desired signal generation principle of the 2-DOF manipulator.

$$
\left\{\begin{array}{l}
\left(M_{11} M_{22}-M_{12} M_{21}\right) \ddot{\theta}_{1}+M_{22} N G_{1}-M_{12} N G_{2}=M_{22} u_{1}-M_{12} u_{2}+M_{22} d_{1}-M_{12} d_{2}, \\
\left(M_{11} M_{22}-M_{12} M_{21}\right) \ddot{\theta}_{2}+M_{11} N G_{2}-M_{21} N G_{1}=-M_{21} u_{1}+M_{11} u_{2}-M_{21} d_{1}+M_{11} d_{2} .
\end{array}\right.
$$

From (16), the descriptions (i)-(ii) are made:

(i) The condition $\Delta=M_{11} M_{22}-M_{12} M_{21}>0$ can be verified according to the definition of the parameters in (1). The control input $u_{1}$ and $u_{2}$ both have an effect on $\theta_{1}$ and $\theta_{2}$. Hence, the two link rods are coupled.

(ii) When the parameters of the system contain uncertain factors and no parameters in (16) are known accurately, the model-based control design will no longer apply. Considering the coupling part of the two link rods as disturbance, the disturbances in each subsystem are estimated and compensated as per the control principle of the designed LADRC. Thus, $\theta_{1}$ and $\theta_{2}$ can be controlled by $u_{1}$ and $u_{2}$, respectively.

3.2. Controller Design. From (16), we have

$$
\left\{\begin{array}{l}
\ddot{\theta}_{1}=-\frac{M_{22} N G_{1}-M_{12} N G_{2}-M_{12} u_{2}+M_{22} d_{1}-M_{12} d_{2}}{\left(M_{11} M_{22}-M_{12} M_{21}\right)} \\
+\left(\frac{M_{22}}{\left(M_{11} M_{22}-M_{12} M_{21}\right)}-b_{1}\right) u_{1}+b_{1} u_{1}, \\
\ddot{\theta}_{2}=-\frac{M_{11} N G_{2}-M_{21} N G_{1}-M_{21} u_{1}-M_{21} d_{1}+M_{11} d_{2}}{\left(M_{11} M_{22}-M_{12} M_{21}\right)} \\
+\left(\frac{M_{11}}{\left(M_{11} M_{22}-M_{12} M_{21}\right)}-b_{2}\right) u_{2}+b_{2} u_{2} .
\end{array}\right.
$$

Assuming the total disturbances of rod 1 and rod 2 as

$$
\begin{aligned}
f_{1}\left(\theta, u_{1}, u_{2}, d_{1}, d_{2}\right)= & \frac{-M_{22} N G_{1}+M_{12} N G_{2}}{\left(M_{11} M_{22}-M_{12} M_{21}\right)}-\frac{M_{12} u_{2}-M_{22} d_{1}+M_{12} d_{2}}{\left(M_{11} M_{22}-M_{12} M_{21}\right)} \\
& +\left(\frac{M_{22}}{\left(M_{11} M_{22}-M_{12} M_{21}\right)}-b_{1}\right) u_{1}, \\
f_{2}\left(\theta, u_{1}, u_{2}, d_{1}, d_{2}\right)= & -\frac{M_{11} N G_{2}-M_{21} N G_{1}}{\left(M_{11} M_{22}-M_{12} M_{21}\right)}-\frac{M_{21} u_{1}+M_{21} d_{1}-M_{11} d_{2}}{\left(M_{11} M_{22}-M_{12} M_{21}\right)} \\
& +\left(\frac{M_{11}}{\left(M_{11} M_{22}-M_{12} M_{21}\right)}-b_{2}\right) u_{2},
\end{aligned}
$$$$
\left\{\ddot{\xi}=f(\xi, w)+b_{1} u_{1}, \ddot{\varsigma}=g(\varsigma, m)+b_{2} u_{2},\right.
$$

(17) can be rewritten as

$$
\left\{\ddot{\theta}_{1}=f_{1}\left(\theta, u_{2}, d_{1}, d_{2}\right)+b_{1} u_{1}, \ddot{\theta}_{2}=f_{2}\left(\theta, u_{1}, d_{1}, d_{2}\right)+b_{2} u_{2} .\right.
$$

(19) is then rewritten as where $w$ is the sum of $d_{1}$ and $d_{2}$ and the coupling control $u_{2}, m$ is the sum of $d_{1}$ and $d_{2}$ and the coupling control $u_{1}$, and $b_{1}$ and $b_{2}$ are the nominal values of the control gain of rod 1 and $\operatorname{rod} 2$, respectively. Assuming $f$ and $g$ are differentiable and that $h=\dot{f}$ and $n=\dot{g},(20)$ can be written in an augmented state-space form: 


$$
\left\{\begin{array}{l}
\dot{\xi}_{1}=\xi_{2}, \\
\dot{\xi}_{2}=\xi_{3}+b_{1} u_{1}, \\
\dot{\xi}_{3}=h(\xi, w), \\
y=\xi_{1}, \\
\dot{\zeta}_{1}=\varsigma_{2}, \\
\dot{\zeta}_{2}=\varsigma_{3}+b_{2} u_{2}, \\
\dot{\zeta}_{3}=n(\varsigma, m), \\
z=\varsigma_{1},
\end{array}\right.
$$

where $\xi=\left[\begin{array}{lll}\xi_{1} & \xi_{2} & \xi_{3}\end{array}\right]^{T} \in \mathbb{R}^{3}, \varsigma=\left[\begin{array}{lll}\varsigma_{1} & \varsigma_{2} & \varsigma_{3}\end{array}\right]^{T} \in \mathbb{R}^{3}$ are the states of the system, and $u_{1}, u_{2} \in R, y, z \in R$ are the input and output of the system, respectively.

LADRC is designed for (21) and the LESO is given as

$$
\left\{\begin{array}{l}
\dot{\vec{\xi}}_{1}=\widehat{\xi}_{2}+\omega_{0} \alpha_{1}\left(\xi_{1}-\widehat{\xi}_{1}\right), \\
\dot{\vec{\xi}}_{2}=\widehat{\xi}_{3}+\omega_{0}^{2} \alpha_{2}\left(\xi_{1}-\widehat{\xi}_{1}\right)+b_{1} u_{1}, \\
\dot{\vec{\xi}}_{3}=\omega_{0}^{3} \alpha_{3}\left(\xi_{1}-\widehat{\xi}_{1}\right), \\
\dot{\widehat{\varsigma}}_{1}=\widehat{\varsigma}_{2}+\omega_{1} \alpha_{1}\left(\varsigma_{1}-\widehat{\varsigma}_{1}\right), \\
\dot{\widehat{\varsigma}}_{2}=\widehat{\varsigma}_{3}+\omega_{1}^{2} \alpha_{2}\left(\varsigma_{1}-\widehat{\varsigma}_{1}\right)+b_{2} u_{2}, \\
\dot{\widehat{\varsigma}}_{3}=\omega_{1}^{3} \alpha_{3}\left(\varsigma_{1}-\widehat{\varsigma}_{1}\right),
\end{array}\right.
$$

where $\omega_{0}, \omega_{1}>0$ and $\alpha_{1}, \alpha_{2}$, and $\alpha_{3}$ are selected such that the characteristic polynomial $s^{3}+\alpha_{1} s^{2}+\alpha_{2} s+\alpha_{3}$ is Hurwitz.

Then, the ADRC control law is given as

$$
\left\{\begin{array}{l}
u_{1}=\left(\phi_{2}^{r}(t)+k_{11}\left(r_{1}-\widehat{\xi}_{1}\right)+k_{12}\left(r_{2}-\widehat{\xi}_{2}\right)-\widehat{\xi}_{3}\right) / b_{1}, \\
u_{2}=\left(\phi_{2}^{s}(t)+k_{21}\left(s_{1}-\widehat{\varsigma}_{1}\right)+k_{22}\left(s_{2}-\widehat{\varsigma}_{2}\right)-\widehat{\varsigma}_{3}\right) / b_{2},
\end{array}\right.
$$

where $r$ is the desired signal of $\xi$, $s$ is the desired signal of $\varsigma$, and the values are assigned and bounded. Let

$$
\left\{\begin{array}{l}
\dot{r}_{1}=r_{2} \\
\dot{r}_{2}=\phi_{2}^{r}(t) \\
\dot{s}_{1}=s_{2} \\
\dot{s}_{2}=\phi_{2}^{s}(t)
\end{array}\right.
$$

$\dot{r}_{1}, \dot{r}_{2}, \dot{s}_{1}$, and $\dot{s}_{2}$ are also bounded and $k_{11}, k_{12}, k_{21}$, and $k_{22}$ are the controller gains selected to make $s^{2}+k_{12} s+k_{11}$ and $s^{2}+k_{22} s+k_{21}$ Hurwitz.

\section{Stability Analysis}

4.1. Stability Analysis of LESO. Let $\widetilde{\xi}_{i}=\xi_{i}-\widehat{\xi}_{i}$ and $\widetilde{\varsigma}=\varsigma_{i}-$ $\widehat{\varsigma}_{i}, i=1,2,3$. From (21) and (22), the observer estimation error can be written as

$$
\left\{\begin{array}{l}
\dot{\widetilde{\xi}}_{1}=\dot{\widetilde{\xi}}_{2}-\omega_{0} \alpha_{1} \widetilde{\xi}_{1}, \\
\dot{\widetilde{\xi}}_{2}=\dot{\widetilde{\xi}}_{3}-\omega_{0}^{2} \alpha_{2} \widetilde{\xi}_{1}, \\
\dot{\widetilde{\xi}}_{3}=h(\xi, w)-\omega_{0}^{3} \alpha_{3} \widetilde{\xi}_{1}, \\
\dot{\widetilde{\zeta}}_{1}=\dot{\widetilde{\varsigma}}_{2}-\omega_{1} \alpha_{1} \widetilde{\varsigma}_{1}, \\
\dot{\widetilde{\varsigma}}_{2}=\dot{\widetilde{\varsigma}}_{3}-\omega_{1}^{2} \alpha_{2} \widetilde{\varsigma}_{1}, \\
\dot{\widetilde{\varsigma}}_{3}=n(\varsigma, m)-\omega_{1}^{3} \alpha_{3} \widetilde{\varsigma}_{1} .
\end{array}\right.
$$

Now, let $\varepsilon_{i}=\widetilde{\xi}_{i} / \omega_{0}^{i-1}$ and $\chi_{i}=\widetilde{\varsigma}_{i} / \omega_{1}^{i-1}, \quad i=1,2,3$, and (25) can be rewritten as

$$
\left\{\begin{array}{l}
\dot{\varepsilon}=\omega_{0} A \varepsilon+B \frac{h(\xi, w)}{\omega_{0}^{2}}, \\
\dot{\chi}=\omega_{1} A \chi+B \frac{n(\varsigma, m)}{\omega_{1}^{2}}
\end{array}\right.
$$

where $A=\left[\begin{array}{lll}-\alpha_{1} & 1 & 0 \\ -\alpha_{2} & 0 & 1 \\ -\alpha_{3} & 0 & 0\end{array}\right]$ and $B=\left[\begin{array}{lll}0 & 0 & 1\end{array}\right]^{T}$.

Theorem 1. Assuming $h(\xi, w)$ and $n(\varsigma, m)$ is bounded, there exists constant $\sigma_{i}>0, \rho_{i}>0$ and a finite $T_{1}>0$, such that $\left|\widetilde{\xi}_{i}(t)\right| \leq \sigma_{i},\left|\widetilde{\varsigma}_{i}(t)\right| \leq \rho_{i}, \quad i=1,2,3, \forall t \geq T_{1}>0, \omega_{0}>0$, and $\omega_{1}>0$. Furthermore, $\sigma_{i}=O\left(1 / \omega_{0}^{k}\right)$ and $\rho_{i}=O\left(1 / \omega_{1}^{k}\right)$, for some positive integer $k$.

Proof. Solving (26), it follows that

$$
\left\{\begin{array}{l}
\varepsilon(t)=e^{\omega_{0} A t} \varepsilon(0)+\int_{0}^{t} e^{\omega_{0} A(t-\tau)} B \frac{h(\xi(\tau), w)}{\omega_{0}^{2}} \mathrm{~d} \tau, \\
\chi(t)=e^{\omega_{1} A t} \chi(0)+\int_{0}^{t} e^{\omega_{1} A(t-\tau)} B \frac{n(\chi(\tau), m)}{\omega_{1}^{2}} \mathrm{~d} \tau,
\end{array}\right.
$$

and let

$$
\begin{aligned}
& p(t)=\int_{0}^{t} e^{\omega_{0} A(t-\tau)} B \frac{h(\xi(\tau), w)}{\omega_{0}^{2}} \mathrm{~d} \tau, \\
& q(t)=\int_{0}^{t} e^{\omega_{1} A(t-\tau)} B \frac{n(\varsigma(\tau), m)}{\omega_{1}^{2}} \mathrm{~d} \tau,
\end{aligned}
$$

as $h(\xi(\tau), w)$ and $n(\varsigma(\tau), m)$ be bounded, i.e., $|h(\xi(\tau), w)| \leq \delta,|n(\varsigma(\tau), m)| \leq \delta$, where $\delta$ is a positive constant. For $i=1,2,3$, we have

$$
\begin{aligned}
& \left|p_{i}(t)\right| \leq \frac{\delta}{\omega_{0}^{3}}\left[\left|\left(A^{-1} B\right)_{i}\right|+\left|\left(A^{-1} e^{\omega_{0} A t} B\right)_{i}\right|\right], \\
& \left|q_{i}(t)\right| \leq \frac{\delta}{\omega_{1}^{3}}\left[\left|\left(A^{-1} B\right)_{i}\right|+\left|\left(A^{-1} e^{\omega_{1} A t} B\right)_{i}\right|\right] .
\end{aligned}
$$

For $A$ and $B$ defined in (26) 


$$
\begin{aligned}
& A^{-1}=\left[\begin{array}{ccc}
0 & 0 & -\frac{1}{\alpha_{3}} \\
1 & 0 & -\frac{\alpha_{1}}{\alpha_{3}} \\
0 & 1 & -\frac{\alpha_{2}}{\alpha_{3}}
\end{array}\right], \\
& \left|\left(A^{-1} B\right)_{i}\right| \leq \nu,
\end{aligned}
$$

where $v=\max _{i=2,3}\left\{1 / \alpha_{3}, \alpha_{i-1} / \alpha_{3}\right\}$. As $A$ is Hurwitz, there exists a finite time $T_{1}>0$ such that

$$
\begin{aligned}
& \left|\left[e^{\omega_{0} A t}\right]_{i j}\right| \leq \frac{1}{\omega_{0}^{3}}, \\
& \left|\left[e^{\omega_{1} A t}\right]_{i j}\right| \leq \frac{1}{\omega_{1}^{3}},
\end{aligned}
$$

for all $t \geq T_{1}, i, j=1,2,3$. Hence,

$$
\begin{aligned}
& \left|\left[e^{\omega_{0} A t} B\right]_{i}\right| \leq \frac{1}{\omega_{0}^{3},} \\
& \left|\left[e^{\omega_{1} A t} B\right]_{i}\right| \leq \frac{1}{\omega_{1}^{3},}
\end{aligned}
$$

for all $t \geq T_{1}, i, j=1,2,3$.

Let $A^{-1}=\left[\begin{array}{lll}g_{11} & g_{12} & g_{13} \\ g_{21} & g_{22} & g_{23} \\ g_{31} & g_{32} & g_{33}\end{array}\right], e^{\omega_{0} A t}=\left[\begin{array}{lll}d_{11} & d_{12} & d_{13} \\ d_{21} & d_{22} & d_{23} \\ d_{31} & d_{32} & d_{33}\end{array}\right]$, and $e^{\omega_{1} A t}=\left[\begin{array}{lll}v_{11} & v_{12} & v_{13} \\ v_{21} & v_{22} & v_{23} \\ v_{31} & v_{32} & v_{33}\end{array}\right]$, we have

$$
\begin{aligned}
& \left|\left(A^{-1} e^{\omega_{0} A t} B\right)_{i}\right| \leq \frac{\mu}{\omega_{0}^{3}}, \\
& \left|\left(A^{-1} e^{\omega_{1} A t} B\right)_{i}\right| \leq \frac{\mu}{\omega_{1}^{3}},
\end{aligned}
$$

for all $t \geq T_{1}, i, j=1,2,3$, where $\mu=\max _{i=2,3}\left\{1 / \alpha_{3}, 1+\alpha_{i-1} / \alpha_{3}\right\}$.

From (29), (30), and (33), we obtain

$$
\left|p_{i}(t)\right| \leq \frac{\delta \nu}{\omega_{0}^{3}}+\frac{\delta \mu}{\omega_{0}^{6}} .
$$

From (29), (30), and (33), we obtain

$$
\left|q_{i}(t)\right| \leq \frac{\delta v}{\omega_{1}^{3}}+\frac{\delta \mu}{\omega_{1}^{6}},
$$

for all $t \geq T_{1}, i, j=1,2,3$.

Let $\quad \varepsilon_{\text {sum }}(0)=\left|\varepsilon_{1}(0)\right|+\left|\varepsilon_{2}(0)\right|+\left|\varepsilon_{3}(0)\right| \quad$ and $\chi_{\text {sum }}(0)=\left|\chi_{1}(0)\right|+\left|\chi_{2}(0)\right|+\left|\chi_{3}(0)\right|$, and it follows that

$$
\begin{gathered}
\left|\left[e^{\omega_{0} A t} \varepsilon(0)_{i}\right]\right| \leq \frac{\varepsilon_{\text {sum }}(0)}{\omega_{0}^{3}}, \\
\left|\left[e^{\omega_{1} A t} \chi(0)_{i}\right]\right| \leq \frac{\chi_{\text {sum }}(0)}{\omega_{1}^{3}},
\end{gathered}
$$

for all $t \geq T_{1}, i, j=1,2,3$.

From (27), one has

$$
\begin{aligned}
& \left\{\begin{array}{l}
\left|\varepsilon_{i}(t)\right| \leq\left|\left[e^{\omega_{0} A t} \varepsilon(0)\right]_{i}\right|+\left|p_{i}(t)\right|, \\
\left|\chi_{i}(t)\right| \leq\left|\left[e^{\omega_{1} A t} \chi(0)\right]_{i}\right|+\left|q_{i}(t)\right| .
\end{array}\right. \\
& \text { Let } \quad \tilde{\xi}_{\text {sun }}(0)=\left|\widetilde{\xi}_{1}(0)\right|+\left|\tilde{\xi}_{2}(0)\right|+\left|\tilde{\xi}_{3}(0)\right| \quad \text { and } \\
& \widetilde{\varsigma}_{\text {sum }}(0)=\left|\widetilde{\varsigma}_{1}(0)\right|+\left|\widetilde{\varsigma}_{2}(0)\right|+\left|\widetilde{\varsigma}_{3}(0)\right| . \quad \text { According } \\
& \varepsilon_{i}=\tilde{\xi}_{i} / \omega_{0}^{i-1}, \chi_{i}=\widetilde{\varsigma}_{i} / \omega_{1}^{i-1} \text {, and (34)-(37), we have } \\
& \left\{\begin{array}{l}
\left|\widetilde{\xi}_{i}(t)\right| \leq\left|\frac{\widetilde{\xi}_{\text {sum }}(0)}{\omega_{0}^{3}}\right|+\frac{\delta \nu}{\omega_{0}^{4-i}}+\frac{\delta \mu}{\omega_{0}^{7-i}}=\sigma_{i}, \\
\left|\widetilde{\varsigma}_{i}(t)\right| \leq\left|\frac{\widetilde{\varsigma}_{\text {sum }}(0)}{\omega_{1}^{3}}\right|+\frac{\delta \nu}{\omega_{1}^{4-i}}+\frac{\delta \mu}{\omega_{1}^{7-i}}=\rho_{i},
\end{array}\right.
\end{aligned}
$$

for all $t \geq T_{1}, i, j=1,2,3$. According to (38), the estimation error of LESO (22) is bounded and the upper bound monotonously decreases with the two observers' bandwidth.

4.2. Stability Analysis of LADRC. From (23), we have

$$
\left\{\begin{array}{l}
u_{1}=\left(\phi_{2}^{r}(t)+k_{11}\left(r_{1}-\widehat{\xi}_{1}\right)+k_{12}\left(r_{2}-\widehat{\xi}_{2}\right)-\widehat{\xi}_{3}\right) / b_{1}, \\
u_{2}=\left(\phi_{2}^{s}(t)+k_{21}\left(s_{1}-\widehat{\varsigma}_{1}\right)+k_{22}\left(s_{2}-\widehat{\varsigma}_{2}\right)-\widehat{\varsigma}_{3}\right) / b_{2},
\end{array}\right.
$$

where $k_{11}, k_{12}, k_{21}$, and $k_{22}$ are the controller gains selected to make $s^{2}+k_{12} s+k_{11}$ and $s^{2}+k_{22} s+k_{21}$ Hurwitz. The closed-loop system becomes

$$
\left\{\begin{array}{l}
\ddot{y}=\left(f-\widehat{\xi}_{3}\right)+k_{11}\left(r_{1}-\widehat{\xi}_{1}\right)+k_{12}\left(r_{2}-\widehat{\xi}_{2}\right)+\phi_{2}^{r}(t), \\
\ddot{z}=\left(g-\widehat{\varsigma}_{3}\right)+k_{21}\left(s_{1}-\widehat{\varsigma}_{1}\right)+k_{22}\left(s_{2}-\widehat{\varsigma}_{2}\right)+\phi_{2}^{s}(t) .
\end{array}\right.
$$

Consider

$$
\dot{\eta}(t)=N \eta(t)+g(t),
$$

where $\eta(t)=\left[\eta_{1}(t), \eta_{2}(t)\right]^{T} \in \mathbb{R}^{2}, g(t)=\left[g_{1}(t), g_{2}(t)\right]^{T} \in$ $\mathbb{R}^{2}$, and $N$ is a $2 \times 2$ matrix.

Lemma 1. If $N$ is Hurwitz and $\lim _{t \rightarrow \infty}\|g(t)\|=0$, then $\lim _{t \rightarrow \infty}\|\eta(t)\|=0$; Zheng et al. [32] provided detailed proof for the lemma.

Theorem 2. Assuming $h(\xi, w)$ and $n(\varsigma, m)$ is bounded, there exist $\lambda_{i}>0, \beta_{i}>0$ and a finite time $T_{5}>0$ such that $\left|e_{i}(t)\right| \leq \lambda_{i},\left|\bar{e}_{i}(t)\right| \leq \beta_{i}, \quad i=1,2, \forall t \geq T_{5}>0, \omega_{0}>0, \omega_{1}>0$, $\omega_{c 1}>0, \omega_{c 2}>0$. Furthermore, $\rho_{i}=O\left(1 / \omega_{c 1}^{j}\right)$ and $\beta_{i}=O\left(1 / \omega_{c 2}^{j}\right), \quad j \in+N$, and $e_{i}=r_{i}-\xi_{i}$ and $\bar{e}_{i}=s_{i}-\varsigma_{i}$, $i=1,2$.

Proof. From (23), we have

$$
\left\{\begin{array}{l}
u_{1}=\left[k_{11}\left(e_{1}+\widetilde{\xi}_{1}\right)+k_{12}\left(e_{2}+\widetilde{\xi}_{2}\right)-\left(\xi_{3}-\widetilde{\xi}_{3}\right)+\phi_{2}^{r}(t)\right] / b_{1}, \\
u_{2}=\left[k_{21}\left(\bar{e}_{1}+\widetilde{\varsigma}_{1}\right)+k_{22}\left(\bar{e}_{2}+\widetilde{\varsigma}_{2}\right)-\left(\varsigma_{3}-\widetilde{\varsigma}_{3}\right)+\phi_{2}^{s}(t)\right] / b_{2},
\end{array}\right.
$$

and it follows that 


$$
\left\{\begin{array}{l}
\dot{e}_{1}=\dot{r}_{1}-\dot{\xi}_{1}=r_{2}-\xi_{2}=e_{2}, \\
\dot{e}_{2}=\dot{r}_{2}-\dot{\xi}_{2}=r_{3}-\left(\xi_{3}+b_{1} u_{1}\right), \\
=-k_{11}\left(e_{1}+\widetilde{\xi}_{1}\right)-k_{12}\left(e_{2}+\widetilde{\xi}_{2}\right)-\widetilde{\xi}_{3} . \\
\dot{\bar{e}}_{1}=\dot{s}_{1}-\dot{\zeta}_{1}=\dot{s}_{2}-\varsigma_{2}=\bar{e}_{2}, \\
\dot{\bar{e}}_{2}=\dot{s}_{2}-\dot{\varsigma}_{2}=\dot{s}_{3}-\left(\varsigma_{3}+b_{2} u_{2}\right), \\
=-k_{21}\left(\bar{e}_{1}+\widetilde{\varsigma}_{1}\right)-k_{22}\left(\bar{e}_{2}+\widetilde{\varsigma}_{2}\right)-\widetilde{\varsigma}_{3} .
\end{array}\right.
$$

Let $e=\left[\begin{array}{ll}e_{1} & e_{2}\end{array}\right]^{T}, \tilde{\xi}=\left[\begin{array}{lll}\widetilde{\xi}_{1} & \widetilde{\xi}_{2} & \widetilde{\xi}_{3}\end{array}\right]^{T}, \bar{e}=\left[\begin{array}{ll}\bar{e}_{1} & \bar{e}_{2}\end{array}\right]^{T}$, and $\widetilde{\varsigma}=\left[\begin{array}{lll}\widetilde{\varsigma}_{1} & \widetilde{\varsigma}_{2} & \widetilde{\varsigma}_{3}\end{array}\right]^{T} ;$ then,

$$
\left\{\begin{array}{l}
\dot{e}(t)=A_{e} e(t)+A_{\tilde{\xi}} \widetilde{\xi}(t) \\
\dot{\bar{e}}(t)=A_{\bar{e}} \bar{e}(t)+A_{\widetilde{\varsigma}} \widetilde{\varsigma}(t)
\end{array}\right.
$$

where

$$
\begin{aligned}
& A_{e}=\left[\begin{array}{cc}
0 & 1 \\
-k_{11} & -k_{12}
\end{array}\right], \\
& A_{\tilde{\xi}}=\left[\begin{array}{ccc}
0 & 0 & 0 \\
-k_{11} & -k_{12} & -1
\end{array}\right], \\
& A_{\bar{e}}=\left[\begin{array}{cc}
0 & 1 \\
-k_{21} & -k_{22}
\end{array}\right], \\
& A_{\widetilde{\zeta}}=\left[\begin{array}{ccc}
0 & 0 & 0 \\
-k_{21} & -k_{22} & -1
\end{array}\right],
\end{aligned}
$$

since $k_{11}, k_{12}, k_{21}$, and $k_{22}$ are selected such that the characteristic polynomials $s^{2}+k_{12} s+k_{11}$ and $s^{2}+k_{22} s+k_{21}$ are Hurwitz and $A_{e}$ and $A_{\bar{e}}$ are Hurwitz. For tunning simplicity, we let $s^{2}+k_{12} s+k_{11}=\left(s+\omega_{c 1}\right)^{2}$ and $s^{2}+k_{22} s+k_{21}=$ $\left(s+\omega_{c 2}\right)^{2}$, where $\omega_{c 1}>0, \omega_{c 2}>0, k_{11}=\omega_{c 1}^{2}, k_{12}=2 \omega_{c 2}, k_{21}=$ $\omega_{c 2}^{2}$, and $k_{22}=2 \omega_{c 2}$ [32]. This implies that $\omega_{c 1}$ and $\omega_{c 2}$, which are controller bandwidths of rod 1 and rod 2, respectively, the only tunning parameters to be adjusted for the controller. obtain

Solving the equation $\dot{e}(t)=A_{e} e(t)+A_{\widetilde{\xi}} \widetilde{\xi}(t)$ in (44), we

$$
e(t)=e^{A e t} e(0)+\int_{0}^{t} e^{A e(t-\tau)} A_{\widetilde{\xi}} \widetilde{\xi}(\tau) \mathrm{d} \tau
$$

According to the equation $\dot{e}(t)=A_{e} e(t)+A_{-} \widetilde{\xi}(t)$ and Theorem 1, we have

$$
\begin{aligned}
& {[A-\widetilde{\xi}(\tau)]_{i=1}=0,} \\
& \left|\left[A_{\tilde{\xi}} \widetilde{\xi}(\tau)\right]_{2}\right| \leq k_{d 1} \sigma_{i}=\gamma, \quad \forall t>T_{1},
\end{aligned}
$$

where $k_{d 1}=1+k_{11}+k_{12}$.

Defining $\quad \Psi=\left[\begin{array}{lllll}0 & 0 & \cdots & 0 & \gamma\end{array}\right]^{T}$ and letting $\varphi(t)=\int_{0}^{t} e^{A e(t-\tau)} A_{\tilde{\xi}} \widetilde{\xi}(\tau) d \tau$, it follows that

$$
\begin{aligned}
\left|\varphi_{i}(t)\right| & \leq\left|\left(A_{e}^{-1} \Psi\right)_{i}\right|+\left|\left(A_{e}^{-1} e^{A_{e} t} \Psi\right)_{i}\right|, \\
\left|\left(A_{e}^{-1} \Psi\right)_{1}\right| & =\frac{\gamma}{k_{11}}=\frac{\gamma}{\omega_{c 1}^{2}} \\
\left|\left(A_{e}^{-1} \Psi\right)_{2}\right| & =0 .
\end{aligned}
$$

Since $A_{e}$ is Hurwitz, there exists a finite time $T_{4}>0$ such that

$$
\left|\left[e^{A_{e} t}\right]_{i j}\right| \leq \frac{1}{\omega_{c 1}^{3}}
$$

for all $t \geq T_{4}, \quad i, j=1,2$.

Let $T_{5}=\max \left\{T_{1}, T_{4}\right\}$. It follows that

$$
\left|\left(e^{A_{e} t} \Psi\right)_{i}\right| \leq \frac{\gamma}{\omega_{c 1}^{3}}
$$

for all $t \geq T_{5}, i=1,2$, and

$$
\left|\left(A_{e}^{-1} e^{A_{e} t} \Psi\right)_{i}\right| \leq\left\{\begin{array}{l}
\left.\frac{1+\sum_{i=2}^{2} k_{1 i}}{\omega_{c 1}^{2}} \frac{\gamma}{\omega_{c 1}^{3}}\right|_{i=1}, \\
\left.\frac{\gamma}{\omega_{c 1}^{3}}\right|_{i=2},
\end{array}\right.
$$

for all $t \geq T_{5}$. From (48) and (51), we obtain

$$
\left|\varphi_{i}(t)\right| \leq\left\{\begin{array}{l}
\frac{\gamma}{\omega_{c 1}^{3}}+\left.\frac{1+\sum_{i=2}^{2} k_{1 i}}{\omega_{c 1}^{2}} \frac{\gamma}{\omega_{c 1}^{3}}\right|_{i=1}, \\
\left.\frac{\gamma}{\omega_{c 1}^{3}}\right|_{i=2},
\end{array}\right.
$$

for all $t \geq T_{5}$. Let $e^{A_{e} t}=\left[\begin{array}{ll}o_{11} & o_{12} \\ o_{21} & o_{22}\end{array}\right], e_{s}(0)=\left|e_{1}(0)\right|+\left|e_{2}(0)\right|$.
It follows that

$$
\left|\left[e^{A_{e} t} e(0)\right]_{i}\right| \leq \frac{e_{s}(0)}{\omega_{c 1}^{3}},
$$

for all $t \geq T_{5}, i=1,2$. From (46), we have

$$
\left|e_{i}(t)\right| \leq\left|\left[e^{A_{e} t} e(0)\right]_{i}\right|+\left|\varphi_{i}(t)\right| .
$$

According to (47) and (52)-(54), we have

$$
\begin{aligned}
& \left|e_{i}(t)\right| \leq\left\{\begin{array}{l}
\frac{e_{s}(0)}{\omega_{c 1}^{3}}+\frac{k_{d 1} \sigma_{i}}{\omega_{c 1}^{2}}+\left.\frac{\left(1+\sum_{i=2}^{2} k_{1 i}\right) k_{d 1} \sigma_{i}}{\omega_{c 1}^{5}}\right|_{i=1}, \\
\left.\frac{e_{s}(0)+k_{d 1} \sigma_{i}}{\omega_{c 1}^{3}}\right|_{i=2},
\end{array}\right. \\
& \leq v
\end{aligned}
$$

for all $t \geq T_{5}, i=1,2$, where

$$
v_{i}=\max \left\{\frac{e_{s}(0)}{\omega_{c 1}^{3}}+\frac{k_{d 1} \sigma_{i}}{\omega_{c 1}^{2}}+\frac{\left(1+\sum_{i=2}^{2} k_{1 i}\right) k_{d 1} \sigma_{i}}{\omega_{c 1}^{5}}, \frac{e_{s}(0)+k_{d 1} \sigma_{i}}{\omega_{c 1}^{3}}\right\} \text {. }
$$

Similarly, we can obtain 


$$
\begin{aligned}
\left|\bar{e}_{i}(t)\right| \leq & \left\{\begin{array}{l}
\frac{\bar{e}_{s}(0)}{\omega_{c 2}^{3}}+\frac{k_{d 2} \zeta_{i}}{\omega_{c 2}^{2}}+\left.\frac{\left(1+\sum_{i=2}^{2} k_{2 i}\right) k_{d 2} \zeta_{i}}{\omega_{c 2}^{5}}\right|_{i=1} \\
\left.\frac{\bar{e}_{s}(0)+k_{d 2} \zeta_{i}}{\omega_{c 2}^{3}}\right|_{i=2}
\end{array}\right. \\
& \leq \beta
\end{aligned}
$$

where

$$
\beta_{i}=\max \left\{\frac{\bar{e}_{s}(0)}{\omega_{c 2}^{3}}+\frac{k_{d 2} \zeta_{i}}{\omega_{c 2}^{2}}+\frac{\left(1+\sum_{i=2}^{2} k_{2 i}\right) k_{d 2} \zeta_{i}}{\omega_{c 2}^{5}}, \frac{\bar{e}_{s}(0)+k_{d 2} \zeta_{i}}{\omega_{c 2}^{3}}\right\} .
$$

According to (55) and (57), we can observe that the error of the desired signal $r_{1}$ and the system output $\xi_{1}$ and the error of desired signal $s_{1}$ and the system output $\varsigma_{1}$ are bounded, and the upper bound monotonously decreases with the two controllers' bandwidth.

Corollary 1. Assuming $h(\xi, w)$ is globally Lipschitz with respect to $\xi$ and $n(\varsigma, m)$ is globally Lipschitz with respect to $\varsigma$, there exist constants $\omega_{0}>0, \omega_{1}>0, \omega_{c 1}>0$, and $\omega_{c 2}>0$, such that the closed-loop system (40) is asymptotically stable.

Proof. From (44), we know

$$
\left\{\begin{array}{l}
\dot{e}(t)=A_{e} e(t)+A_{\tilde{\xi}} \widetilde{\xi}(t) \\
\dot{\bar{e}}(t)=A_{\bar{e}} \bar{e}(t)+A_{\widetilde{\varsigma}} \widetilde{\varsigma}(t)
\end{array}\right.
$$

According to Theorem 1 postulated by [27], $\lim _{t \rightarrow \infty}\left\|A_{\widetilde{\xi}} \widetilde{\xi}(t)\right\|=0$ and $\lim _{t \rightarrow \infty}\left\|A_{\widetilde{\zeta}} \widetilde{\varsigma}(t)\right\|=0$, if $h(\xi, w)$ is globally Lipschitz with respect to $\xi$ and $n(\varsigma, m)$ is globally Lipschitz with respect to $\varsigma$. Furthermore, according to Lemma 1 , since $A_{e}$ and $A_{\bar{e}}$ is Hurwitz and $\lim _{t \rightarrow \infty}$ $\left\|A_{\tilde{\xi}} \widetilde{\xi}(t)\right\|=0, \quad \lim _{t \rightarrow \infty}\left\|A_{\widetilde{\varsigma}} \widetilde{\varsigma}(t)\right\|=0$, such that $\lim _{t \rightarrow \infty}$ $e_{i}(t)=0, \lim _{t \rightarrow \infty} \bar{e}_{i}(t)=0, i=1,2$, the closed-loop system (40) is asymptotically stable.

4.2.1. Simulation. In this section, a simulation study on a 2DOF manipulator is conducted to demonstrate the strong robust and high precision control effect of LADRC. The 3D model of the system is established by SolidWorks and is illustrated in Figure 3. Furthermore, by generating the XML file of the model in SolidWorks and accessing the file in Matlab, we can obtain the Simulink model of the 2-DOF manipulator, as shown in Figure 4. The simulation of control is obtained from Matlab/Simulink.

In the simulation, the LADRC and PID are applied for controlling plant (20) with the same regular octagon trajectory. The controller parameters are listed in Table 1.

4.2.2. Simulation of Increasing Payload. Upon adding a $1.5 \mathrm{~kg}$ payload to the end effector, the controller parameters of LADRC and PID remain constant. The tracking curve and tracking error between LADRC and PID are

compared in Figure 5. From Figures 5(a), 5(c), and 5(e), we can observe that although the payload is increased, LADRC and PID can make the end effector track the octagonal trajectory. However, LADRC has a more accurate tracking performance both in $x$ and $y$ directions. From Figures 5(b) and 5(d), we can observe that the control of LADRC has smaller tracking errors and less tracking error fluctuations.

4.3. Simulation of Sudden Disturbances. When the end effector has no payload, we add step signals of the same amplitude to several angles and edges of the octagonal trajectory, respectively. From Figure 6, we can conclude that compared with PID control, the maximum mutation of the tracking response of the LADRC method is smaller, the response fluctuation is smaller, and the time of the response recovery to the desired trajectory is shorter despite the position of the disturbances in the octagonal trajectory. The tracking errors depicted in Figure 7 also show that when the disturbances occur suddenly, the LADRC method has smaller maximum tracking errors, less tracking deviation fluctuation, and shorter deviation convergence times. Thus, the simulation results show that LADRC has better robustness and better control performance than PID control.

\section{Experiment}

Here, the proposed LADRC is further examined through an experiment, which is conducted on a 2-DOF manipulator, as shown in Figure 8. The system comprises two motors, two reducers, two rods, GTHD servodrivers, and a GT-800-SV motion control card. The nominal values of the system parameters are given in Table 2.

The sampling period in the experiment is set as $0.001 \mathrm{~s}$ and the solver in Matlab/Simulink environment is set as ode $l$ (Euler).

First, the experiment of an increasing payload is conducted and the experiment of sudden disturbances is then conducted without payload, i.e., the step signals of the same amplitude are suddenly added at certain angles and edges of the octagonal trajectory, respectively. The controller parameters in the experiment are listed in Table 3.

5.1. Experiment of Increasing Payload. Upon adding a $1.5 \mathrm{~kg}$ payload to the end effector, the controller parameters of LADRC and PID remain constant. Experimental results are shown in Figure 9. From Figures 9(a), 9(c), and 9(e), we can observe that when the payload is increased, LADRC and PID can also make the end effector track the octagonal trajectory. However, LADRC has a more accurate tracking performance despite its position in $x$ or $y$ directions. Figures 9(b) and $9(\mathrm{~d})$ show that the control of LADRC has smaller tracking errors and less tracking error fluctuations than PID.

5.2. Experiment of Sudden Disturbances. When the end effector has no payload, we add a step signal of $20 \mathrm{~V}$ to the 


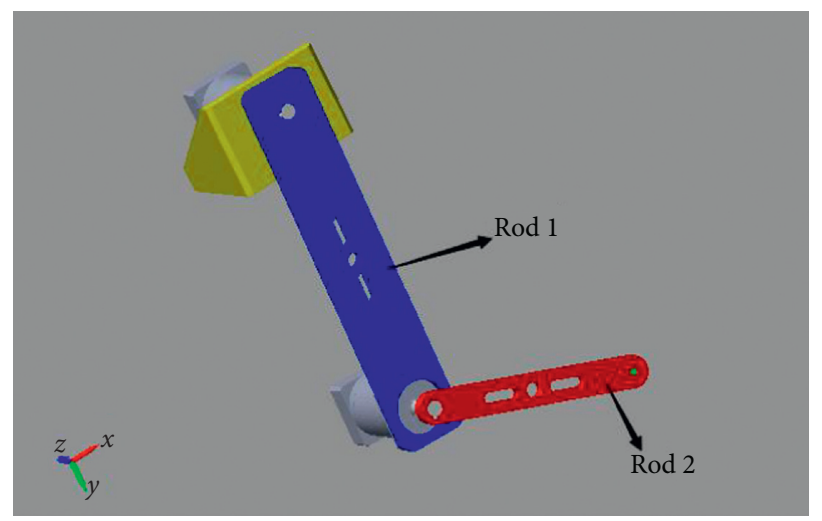

FIGURE 3: 3D model of the 2-DOF manipulator.

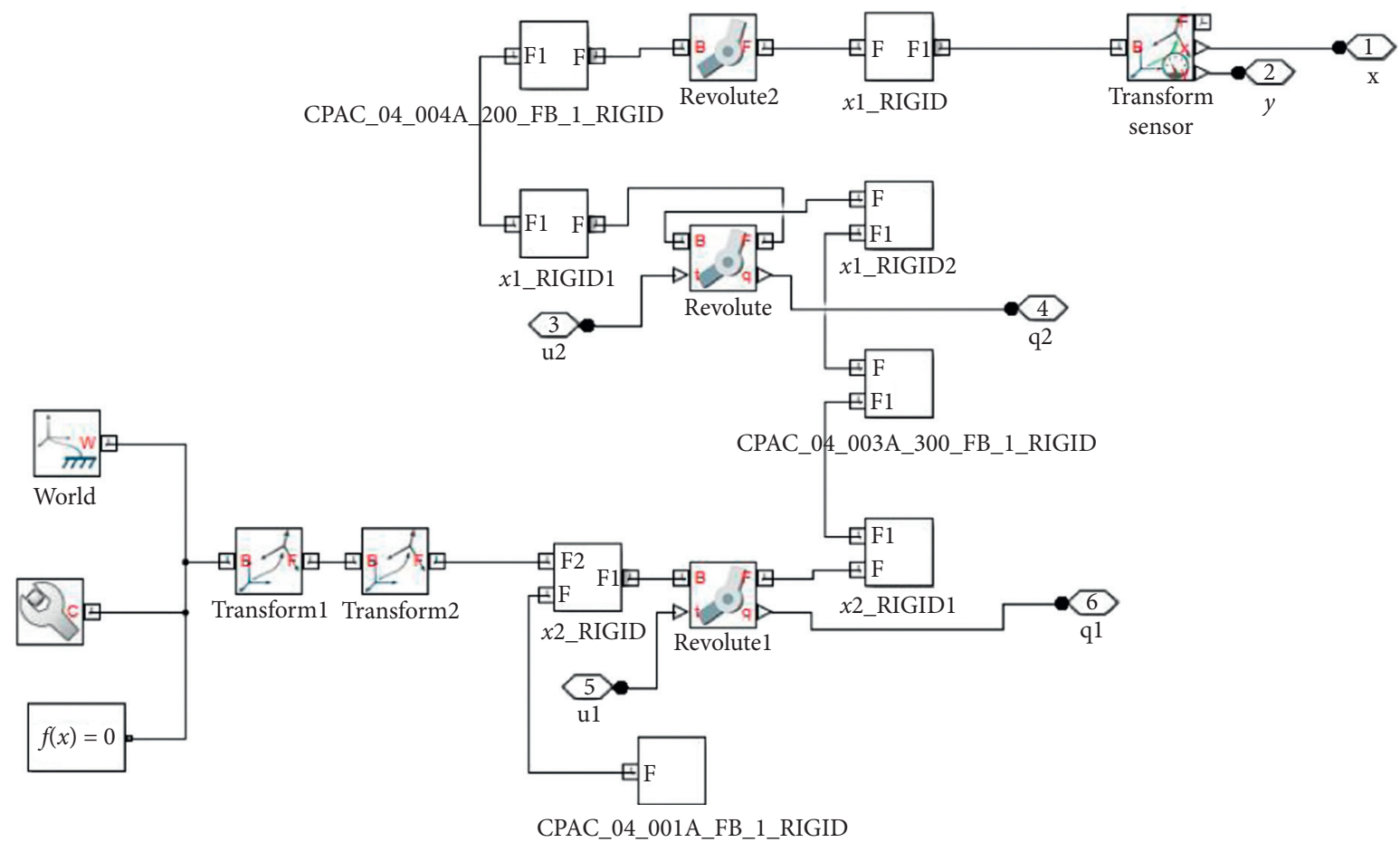

FIgURE 4: Simulink model of the 2-DOF manipulator.

TABLE 1: LADRC and PID controller parameters in simulation.

\begin{tabular}{cccccccccc}
\hline \multicolumn{1}{c}{ LADRC } & & PID \\
\hline \multirow{2}{*}{ Rod 1 } & $\omega_{c 1}$ & $\omega_{0}$ & $b_{1}$ & $\alpha_{1}$ & $\alpha_{2}$ & $\alpha_{3}$ & $k_{p 1}$ & $k_{i 1}$ & $k_{d 1}$ \\
& 35 & 500 & 20 & 3 & 3 & 1 & 300 & 50 & 0.5 \\
\hline \multirow{2}{*}{ Rod 2 } & $\omega_{c 2}$ & $\omega_{1}$ & $b_{2}$ & $\alpha_{1}$ & $\alpha_{2}$ & $\alpha_{3}$ & $k_{p 2}$ & $k_{i 2}$ & $k_{d 2}$ \\
& 30 & 500 & 55 & 3 & 3 & 1 & 150 & 40 & 0.5 \\
\hline
\end{tabular}



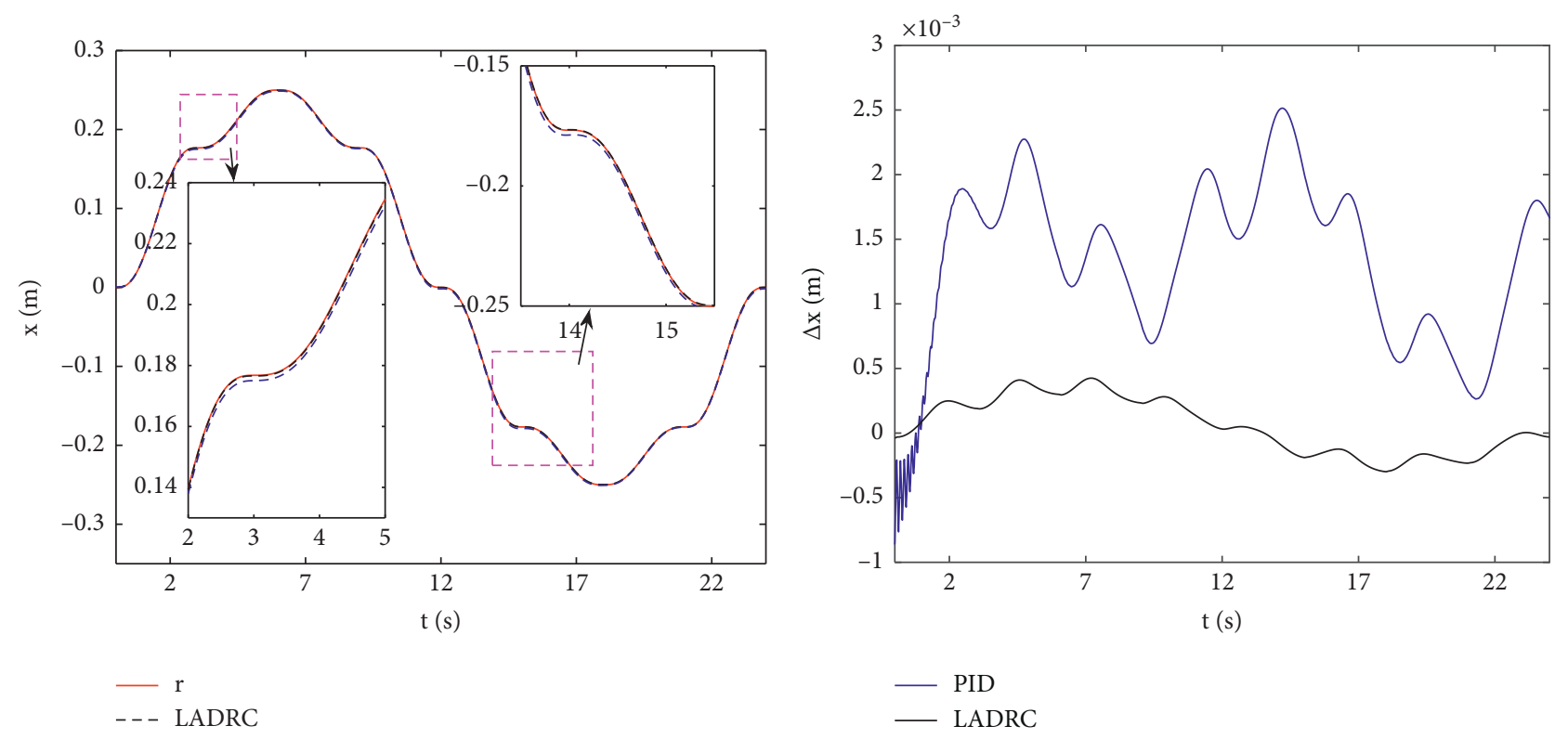

(a)

(b)
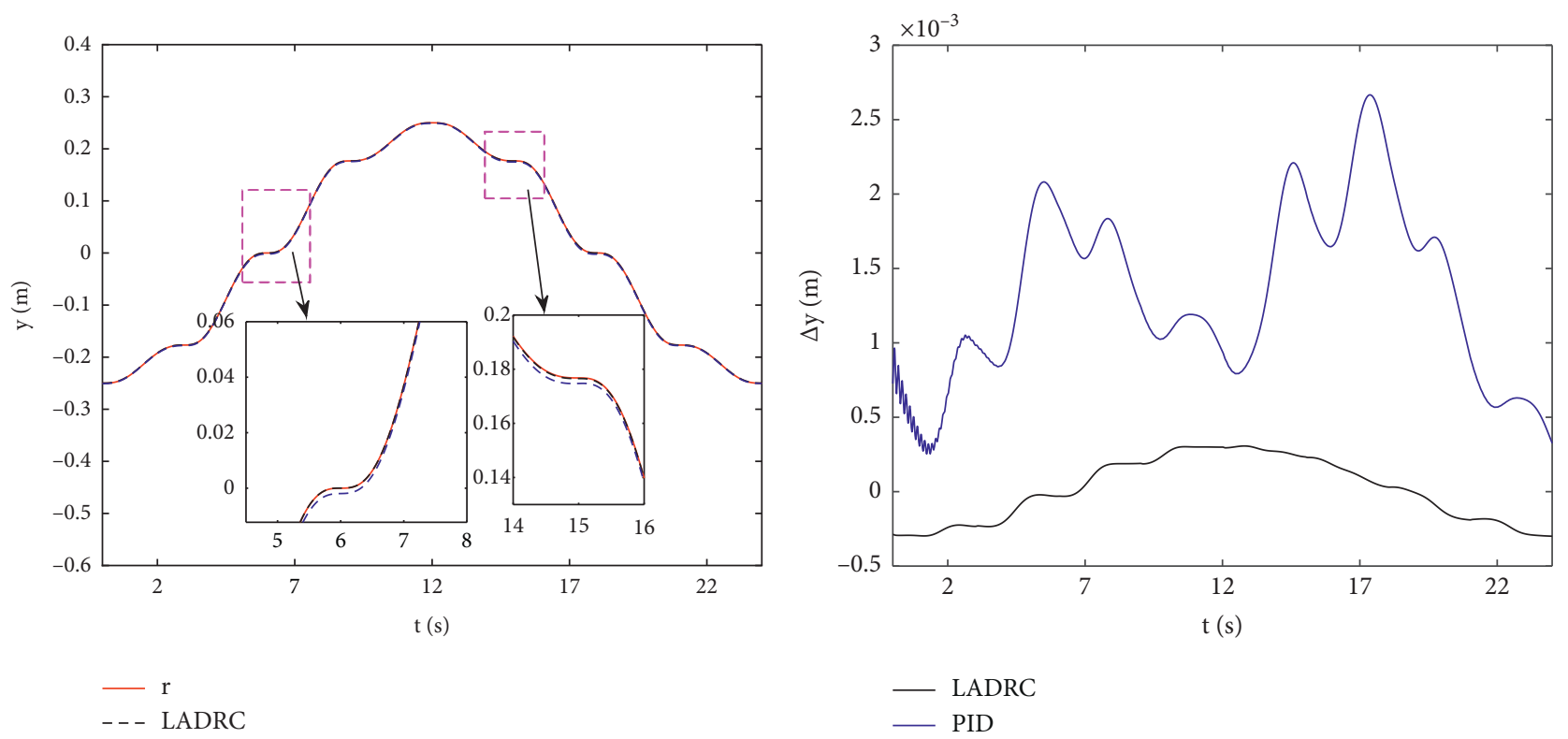

- - LADRC

— PID

(c)

(d)

Figure 5: Continued. 


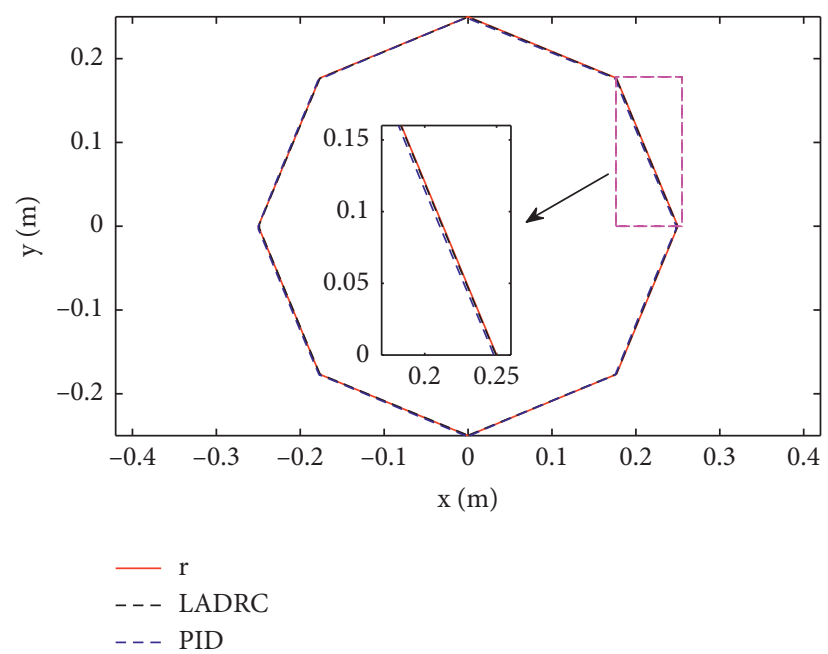

(e)

FIGURE 5: Simulation comparison of tracking performance between LADRC and PID. (a) Comparison of tracking trajectory in the $x$ direction. (b) Comparison of tracking errors in the $x$-direction. (c) Comparison of tracking trajectory in the $y$-direction. (d) Comparison of tracking errors in the $y$-direction. (e) Tracking comparison of regular octagonal trajectory.

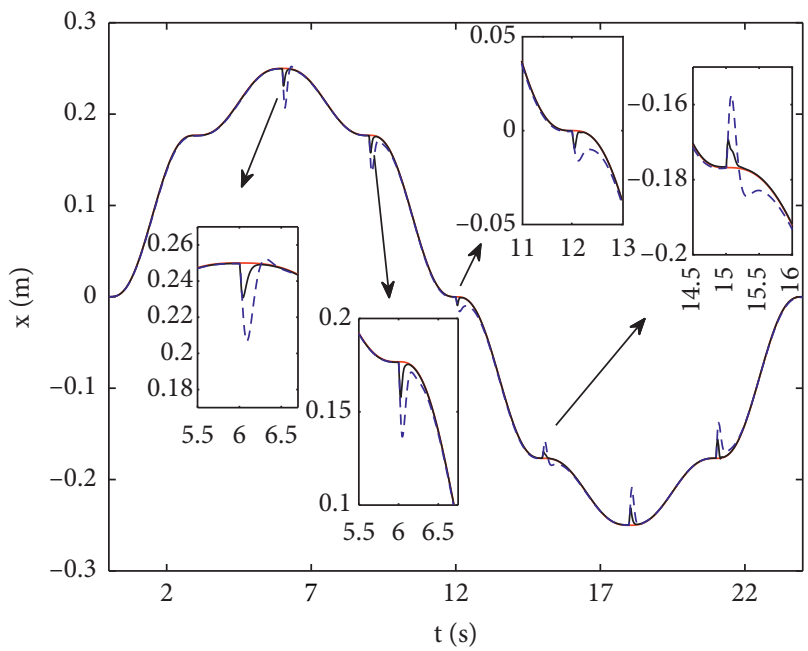

$\begin{array}{ll}- & \mathrm{r} \\ - & \text { LADRC } \\ --- & \text { PID }\end{array}$

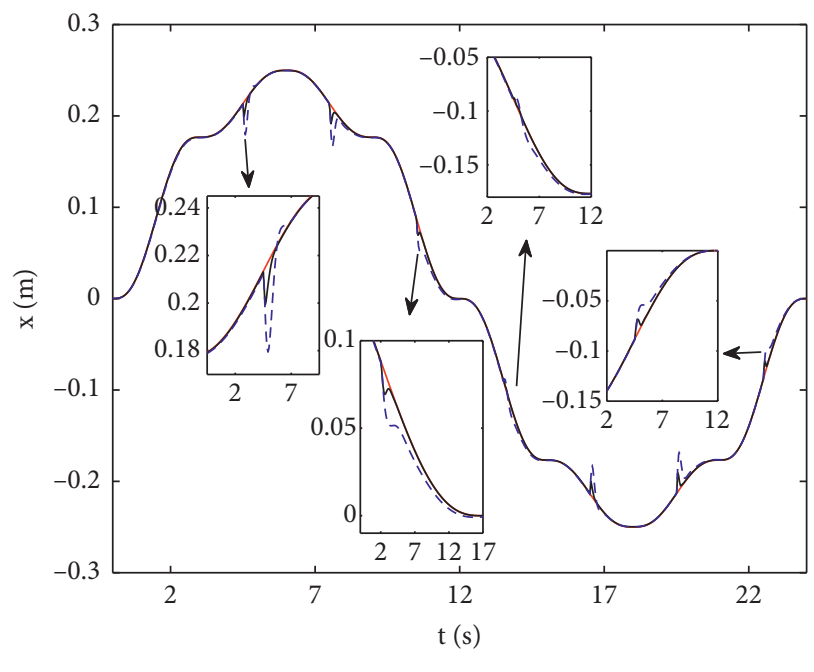

- LADRC

(a)

Figure 6: Continued. 

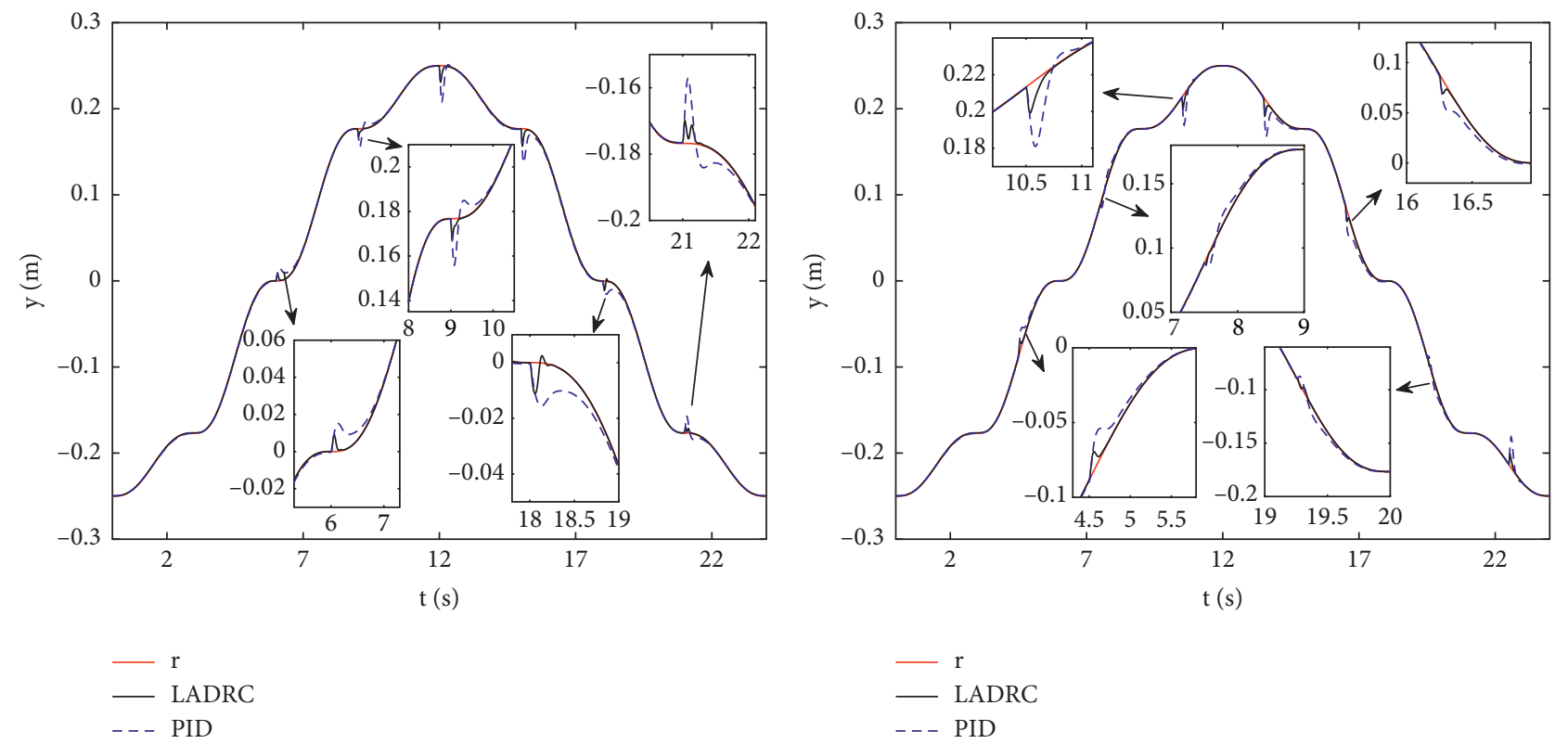

(c)

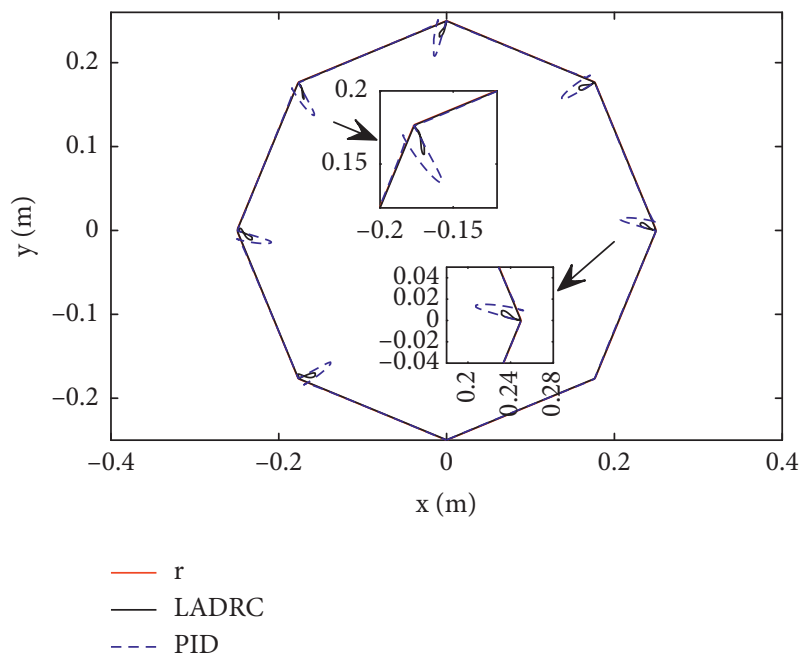

(e)

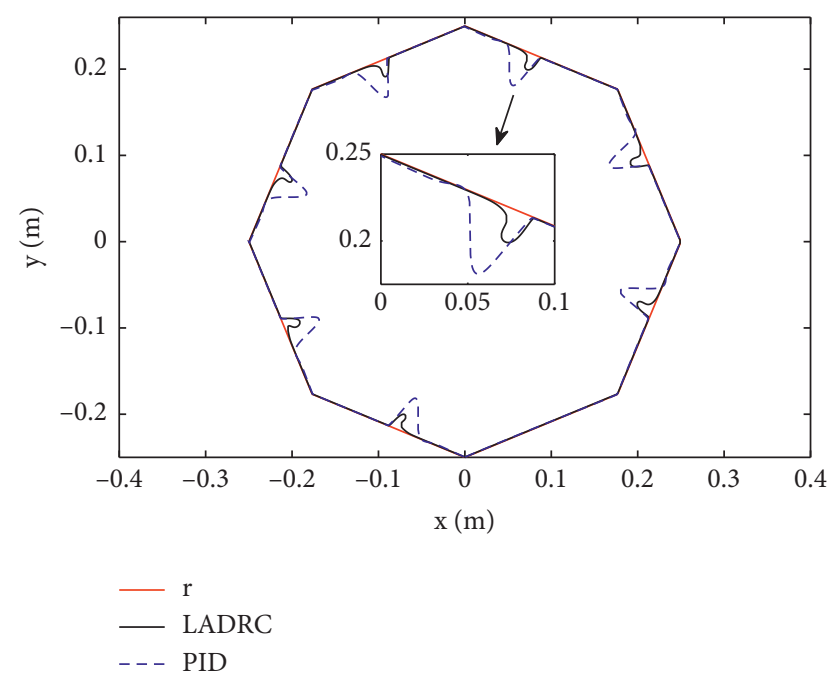

(f)

FIGURE 6: Simulation comparison of tracking performance between LADRC and PID during a sudden disturbance. (a) Comparison of trajectory tracking with disturbance at the angles of octagonal in the $x$-direction. (b) Comparison of trajectory tracking with disturbance at the edges of octagonal in the $x$-direction. (c) Comparison of trajectory tracking with disturbance at the angles of octagonal in the $y$ direction. (d) Comparison of trajectory tracking with disturbance at the edges of octagonal in the $y$-direction. (e) Comparison of trajectory tracking with disturbance at the angles of octagonal. (f) Comparison of trajectory tracking with disturbance at the edges of octagonal.

several angles and edges of the octagonal trajectory. The experimental results are plotted in Figures 10 and 11. From Figure 10, we can observe that despite the position of the disturbances in the octagonal trajectory, the LADRC method has a smaller maximum trajectory deviation, faster response, smaller tracking fluctuations, and shorter tracking error convergence times when compared to PID. The tracking errors depicted in Figure 11 also show that when the disturbances occur suddenly, the LADRC method has smaller maximum tracking errors, less tracking deviation fluctuation, and shorter deviation convergence times. The experimental results verify that the control performance and robustness of LADRC are better than PID. 

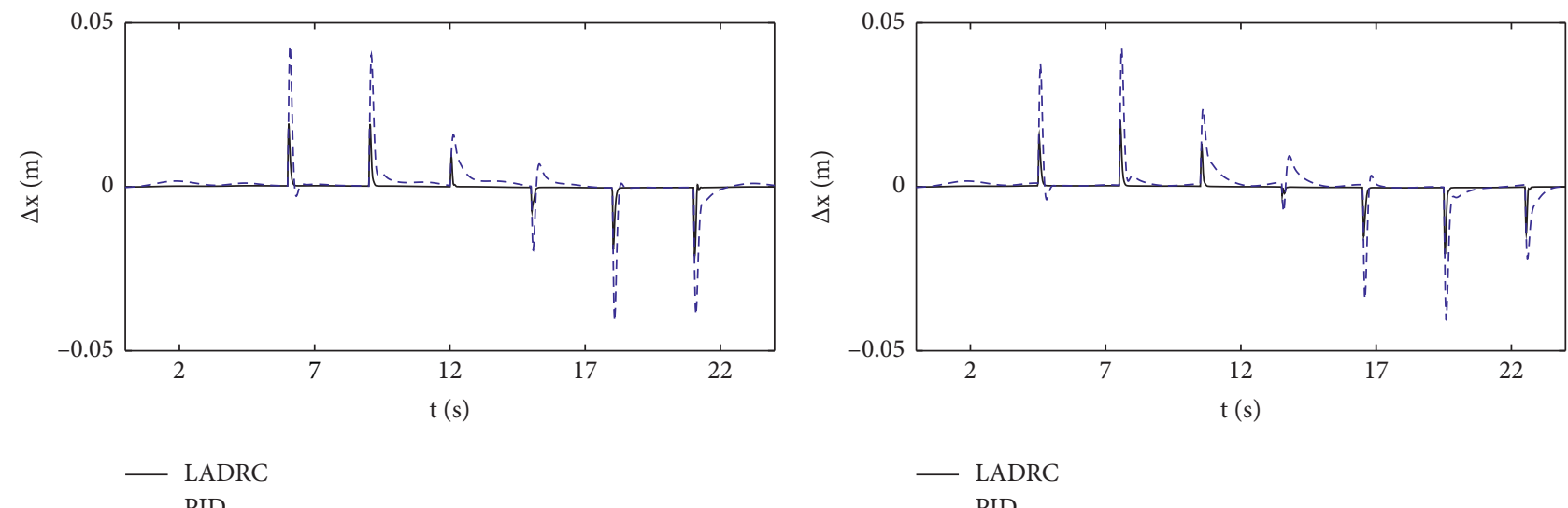

-. - PID

(a)
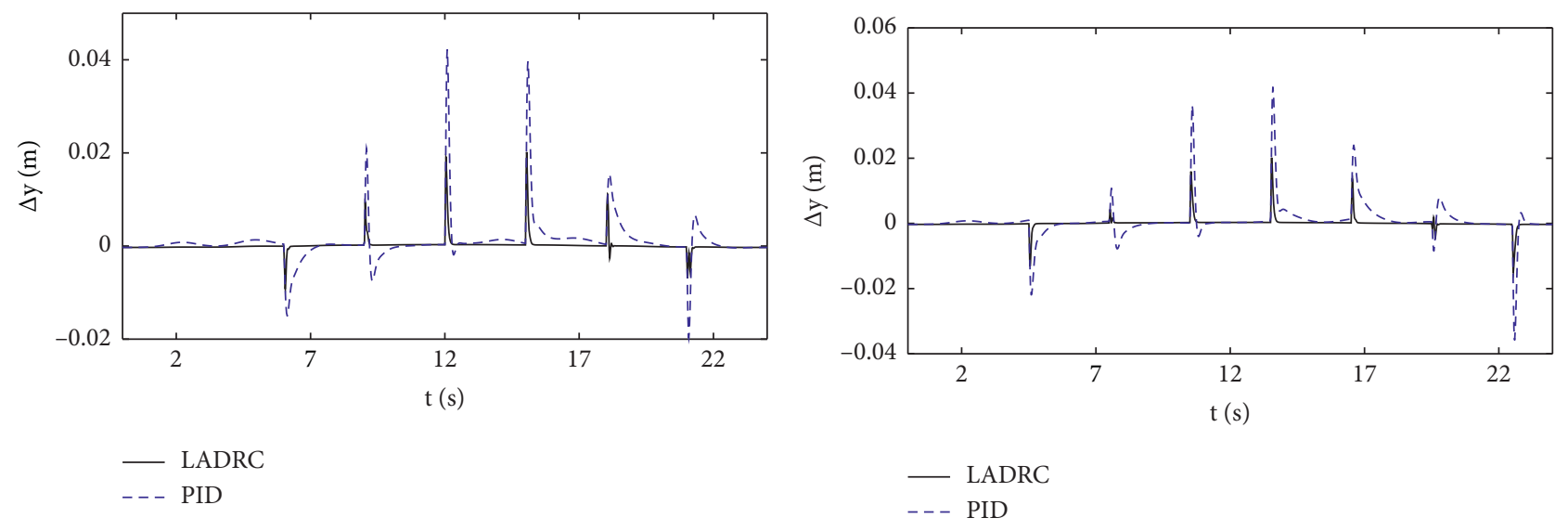

(c)

(d)

FIgURE 7: Simulation comparison of tracking errors between LADRC and PID during sudden disturbance. (a) Comparison of tracking errors with a disturbance at the angles of octagonal in the $x$-direction. (b) Comparison of tracking errors with a disturbance at the edges of octagonal in the $x$-direction. (c) Comparison of tracking errors with disturbance at the angles of octagonal in the $y$-direction. (d) Comparison of tracking errors with disturbance at the edges of octagonal in the $y$-direction.

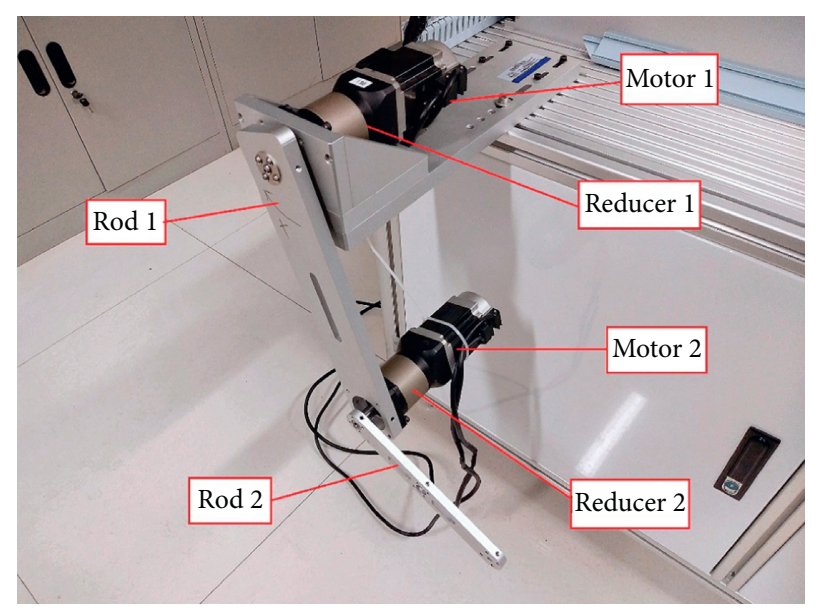

FIGURE 8: Experimental platform of a 2-DOF manipulator. 
TABLE 2: Nominal values of the 2-DOF manipulator system parameters.

\begin{tabular}{lcccc}
\hline Parameters & $m_{1}(\mathrm{~kg})$ & $m_{2}(\mathrm{~kg})$ & $l_{1}(\mathrm{~m})$ & $l_{2}(\mathrm{~m})$ \\
\hline Value & 0.85 & 0.15 & 0.3 & 0.2
\end{tabular}

TABLE 3: LADRC and PID controller parameters in experiment.

\begin{tabular}{cccccccccc}
\hline \multicolumn{1}{c}{ LADRC } & & PID \\
\hline \multirow{2}{*}{ Rod 1 } & $\omega_{c 1}$ & $\omega_{0}$ & $b_{1}$ & $\alpha_{1}$ & $\alpha_{2}$ & $\alpha_{3}$ & $k_{p 1}$ & $k_{i 1}$ & $k_{d 1}$ \\
& 40 & 200 & 900 & 3 & 3 & 1 & 1600 & 50 & 80 \\
\hline \multirow{2}{*}{ Rod 2 } & $\omega_{c 2}$ & $\omega_{1}$ & $b_{2}$ & $\alpha_{1}$ & $\alpha_{2}$ & $\alpha_{3}$ & $k_{p 2}$ & $k_{i 2}$ & $k_{d 2}$ \\
& 50 & 200 & 1000 & 3 & 3 & 1 & 2500 & 50 & 100 \\
\hline
\end{tabular}

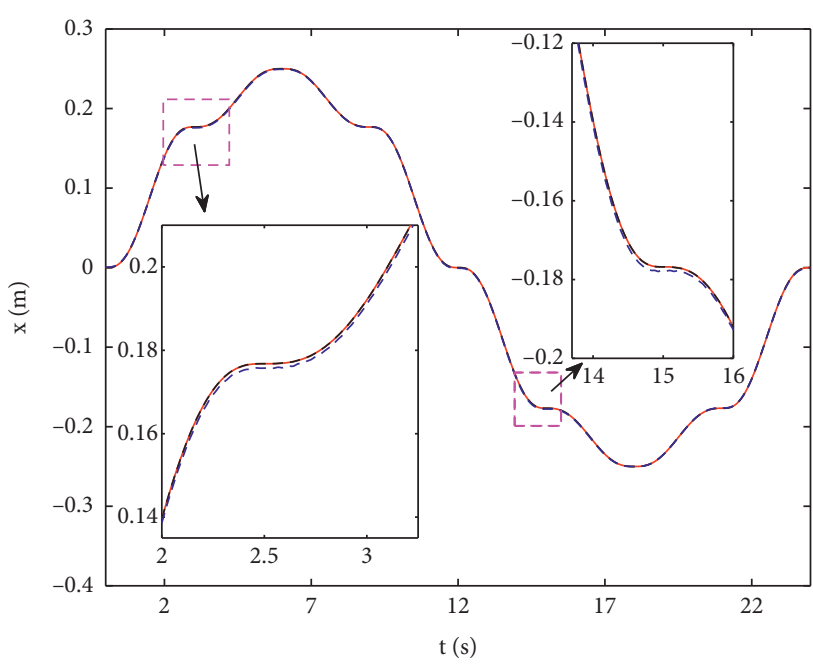

- r

--- LADRC

- - - PID

(a)

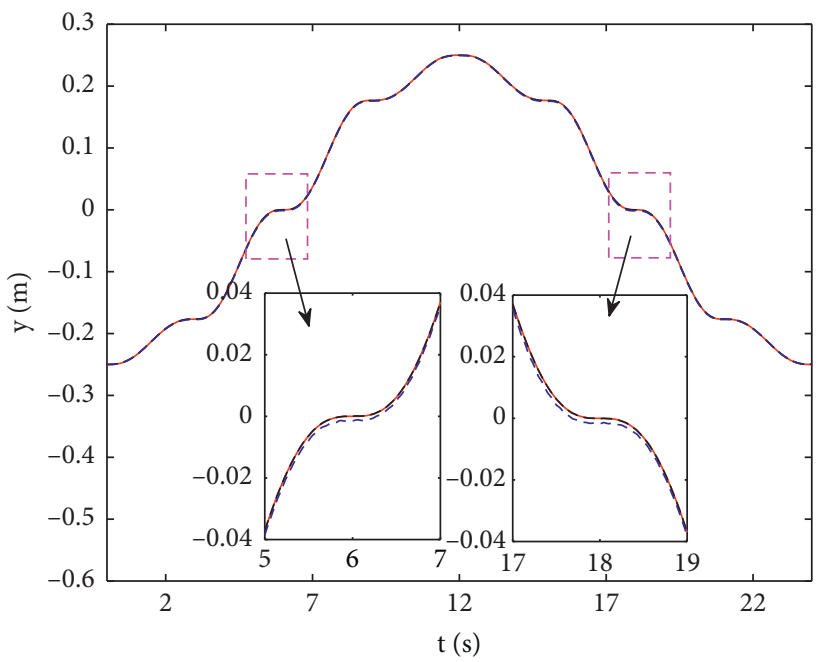

r

- - LADRC

- - - PID

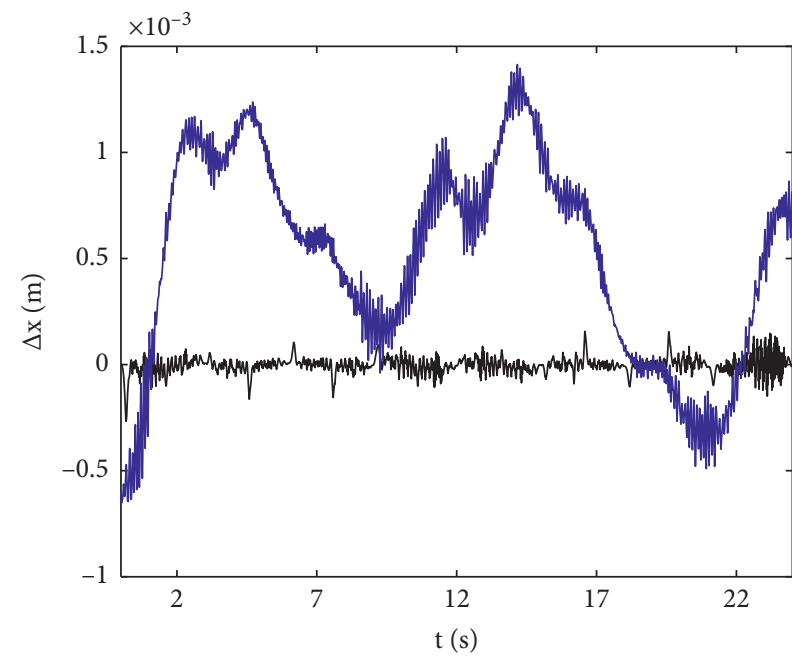

— LADRC

— PID

(b)

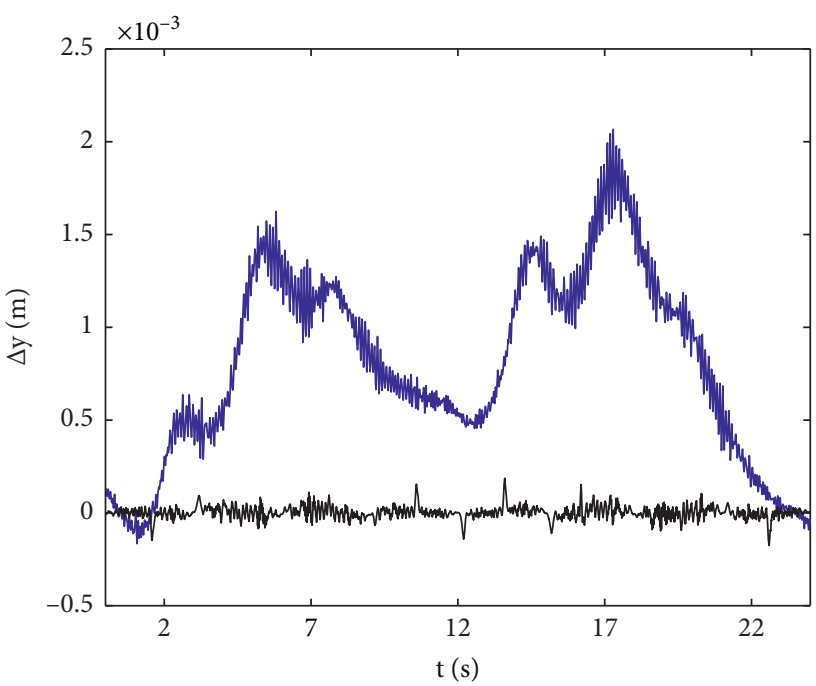

— PID

- LADRC

(c)

FIgURE 9: Continued. 


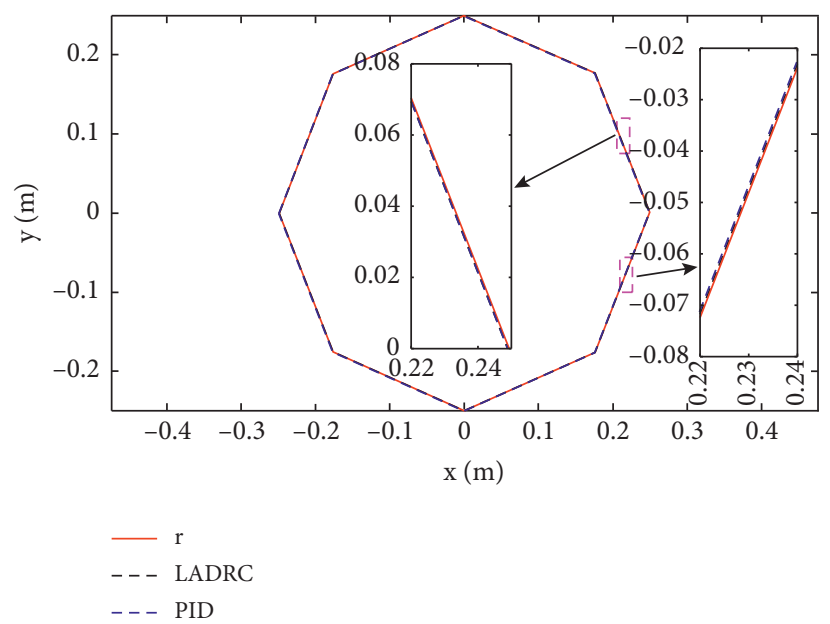

(e)

FIGURE 9: Experimental comparison of tracking performance between LADRC and PID. (a) Comparison of tracking trajectory in the $x$ direction. (b) Comparison of tracking errors in the $x$-direction. (c) Comparison of tracking trajectory in the $y$-direction. (d) Comparison of tracking errors in the $y$-direction. (e) Tracking comparison of regular octagonal trajectory.

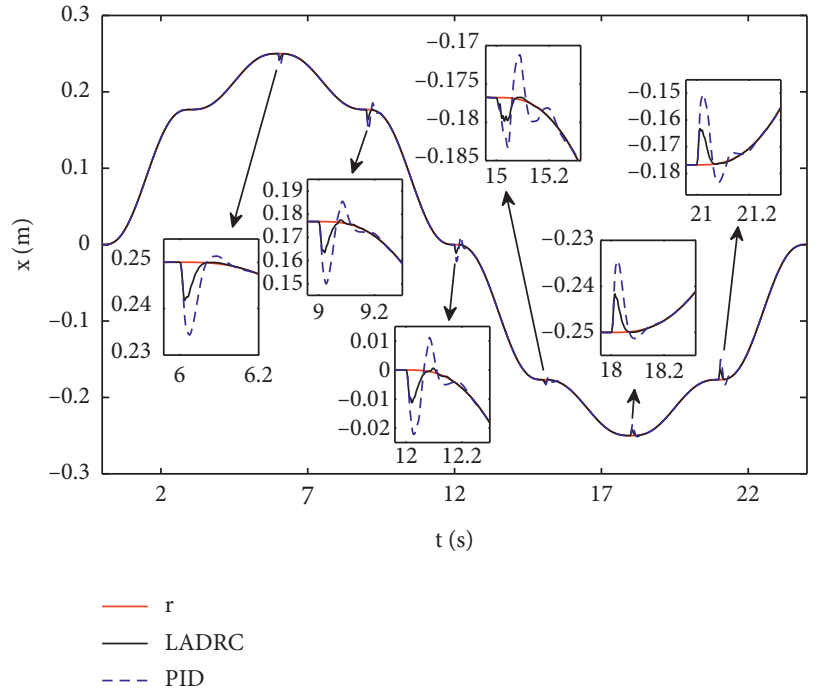

(a)

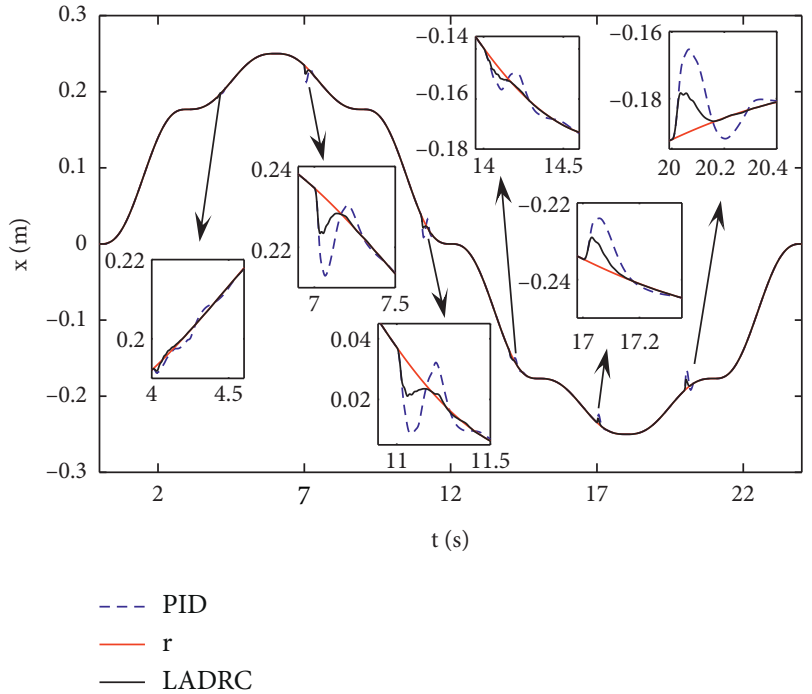

(b)

Figure 10: Continued. 

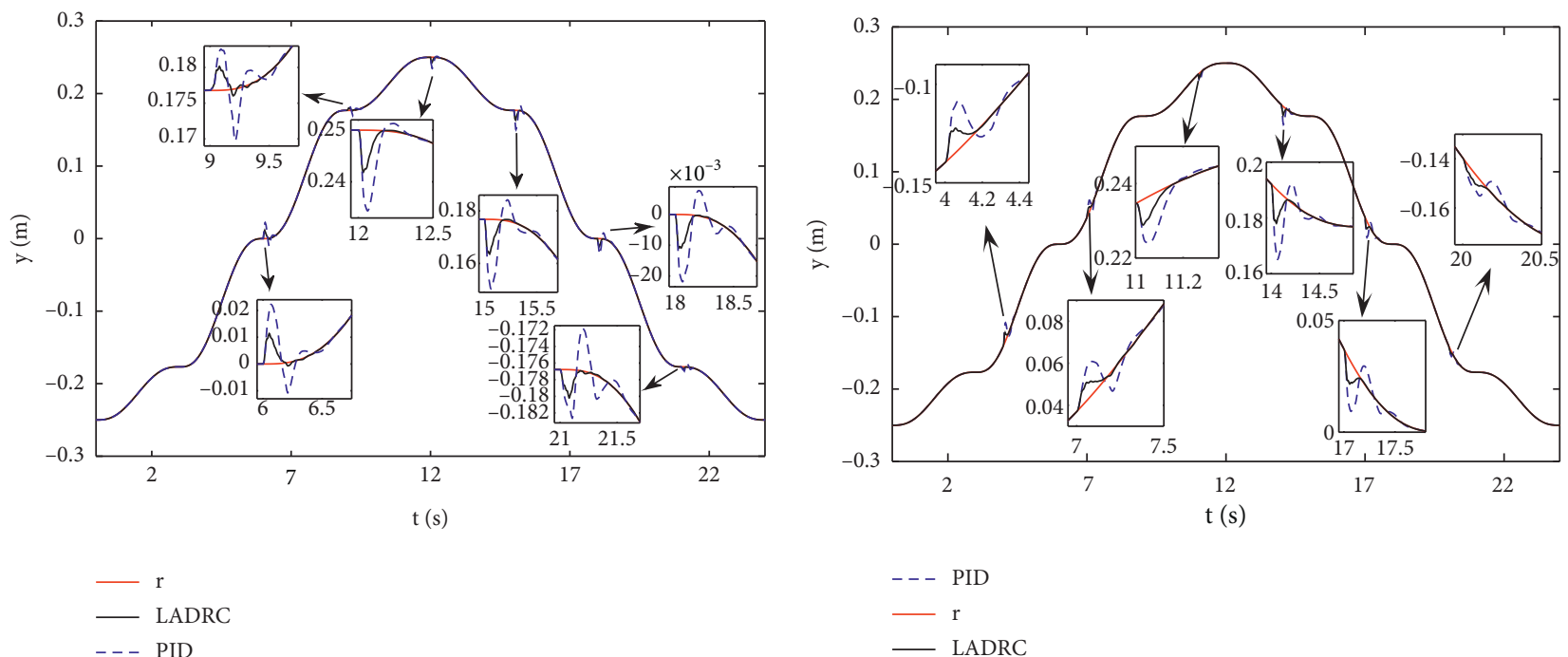

(c)
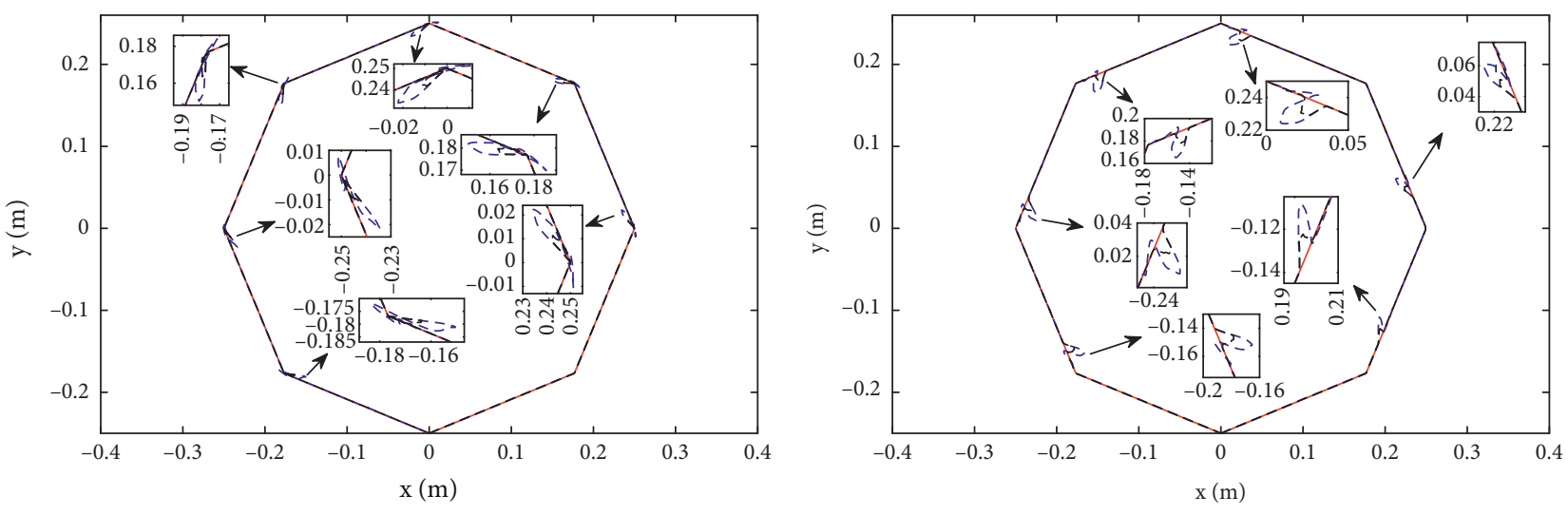

$\begin{array}{ll}- & \mathrm{r} \\ --- & \text { PID } \\ --- & \text { LADRC }\end{array}$

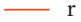

- - - PID

--- LADRC

(e)

(f)

FIGURE 10: Experimental comparison of the tracking performance between LADRC and PID. during sudden disturbance. (a) Comparison of trajectory tracking with disturbance at the angles of octagonal in the $x$-direction. (b) Comparison of trajectory tracking with disturbance at the edges of octagonal in the $x$-direction. (c) Comparison of trajectory tracking with disturbance at the angles of octagonal in the $y$-direction. (d) Comparison of trajectory tracking with disturbance at the edges of octagonal in the $y$ direction (e) Comparison of trajectory tracking with disturbance at the angles of octagonal. (f) Comparison of trajectory tracking with disturbance at the edges of octagonal. 


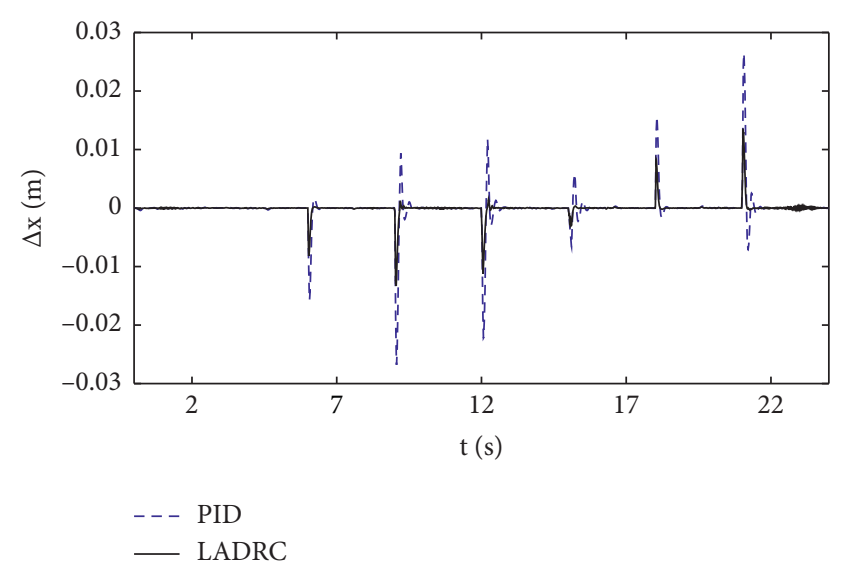

(a)

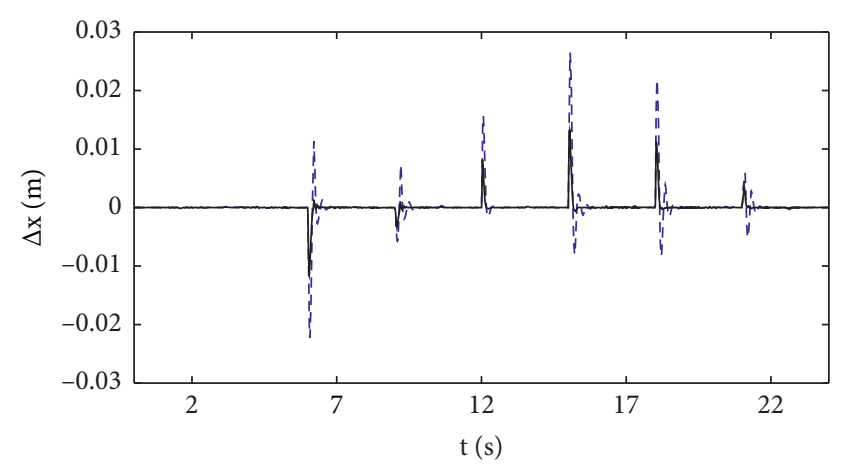

--- PID

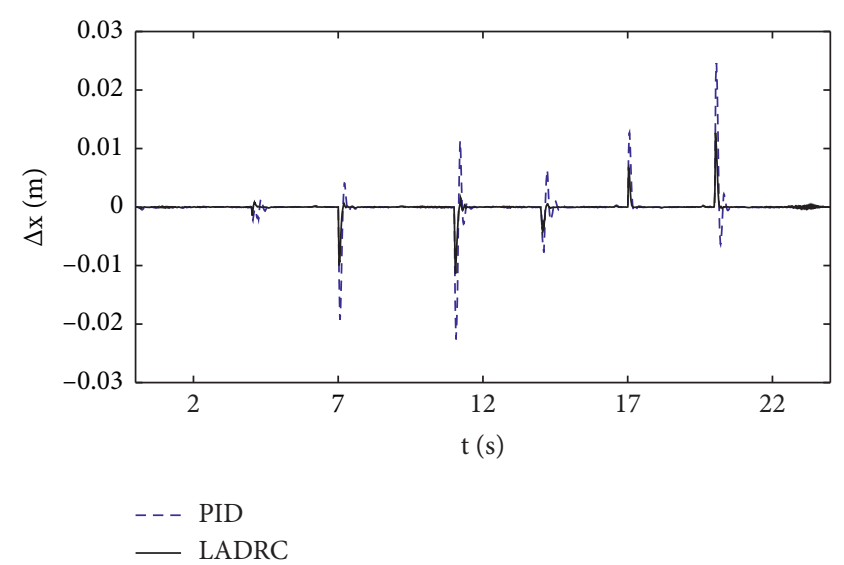

(b)

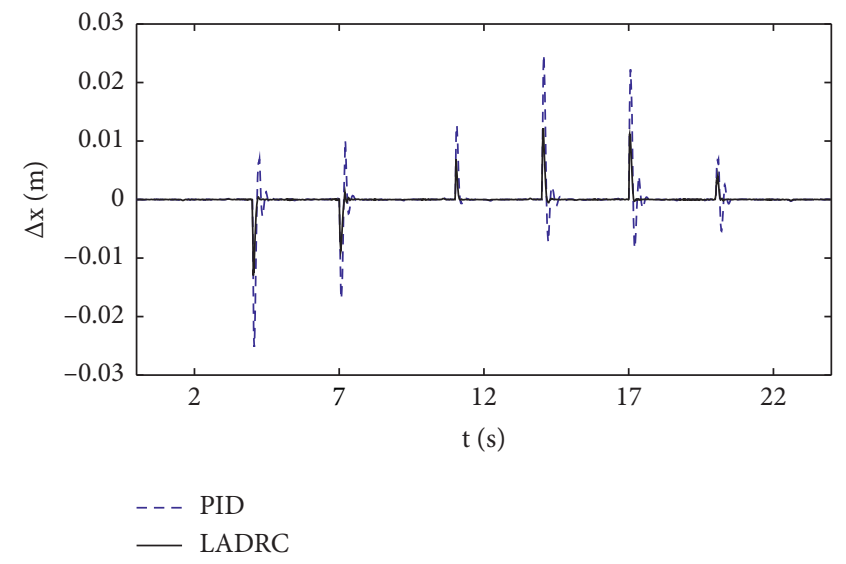

(d)

FIGURE 11: Experimental comparison of tracking errors between LADRC and PID during sudden disturbance. (a) Comparison of tracking errors with disturbance at the angles of octagonal in the $x$-direction. (b) Comparison of tracking errors with disturbance at the edges of octagonal in the $x$-direction. (c) Comparison of tracking errors with disturbance at the angles of octagonal in the $y$-direction. (d) Comparison of tracking errors with disturbance at the edges of octagonal in the $y$-direction.

\section{Conclusions}

In this study, the decoupled mathematical model of the 2DOF manipulator is established and the desired trajectory is planned using the quintic polynomial method. LESO is adopted to estimate the total disturbances comprising dynamic decoupling, internal uncertainties, and external disturbances. Furthermore, a linear state error feedback control law is designed to compensate for the total disturbances and to establish the stability of the closed-loop system. In addition, the stability of the proposed LESO and the closedloop system is analyzed. The relationship between the performance of the closed-loop system and the controller parameters is also demonstrated. Furthermore, the comparative simulations and experiments verify the better performance and stronger robustness of the proposed LADRC than that of PID.

\section{Data Availability}

The data used to support the findings of this study are included within the article.

\section{Conflicts of Interest}

The authors declare that they have no conflicts of interest.

\section{Acknowledgments}

This study was supported by the National Natural Science Foundations of China (1905541) and Young Talent fund of University Association for Science and Technology in Shannxi, China (20190412).

\section{References}

[1] F. L. Lewis, D. M. Dawson, and C. T. Abdallah, "Control of robot manipulators," 1993.

[2] S. S. Ge, T. H. Lee, and C. J. Harris, Adaptive Neural Network Control of Robotic Manipulators, World Science, Singapore, 1998.

[3] Q. Chen, Y. Wang, and H. Chen, "Comparative research of trajectory tracking performance of robotic manipulator based on PD control scheme," Control and Decision, vol. 18, no. 1, pp. 53-57, 2003, in Chinese. 
[4] L. Wang and X. Yang, "Trajectory tracking control for robotic manipulators endowed with feedforward and neural networks," Electric Machines and Control, vol. 8, pp. 117-122, 2013, in Chinese.

[5] T.-Y. Kuc and W.-G. Han, "An adaptive PID learning control of robot manipulators," Automatica, vol. 36, no. 5, pp. 717$725,2000$.

[6] P. R. Ouyang, W. J. Zhang, and M. M. Gupta, “An adaptive switching learning control method for trajectory tracking of robot manipulators," Mechatronics, vol. 16, no. 1, pp. 51-61, 2006.

[7] T. T. Tran, G. E. S. Sam, and H. E. Wei, "Adaptive control for an uncertain robotic manipulator with input saturations," Control Theory and Technology, vol. 14, p. 2, 2016.

[8] R.-J. Wai and P.-C. Chen, "Robust neural-fuzzy-network control for robot manipulator including actuator dynamics," IEEE Transactions on Industrial Electronics, vol. 53, no. 4, pp. 1328-1349, 2006.

[9] L. Lin, H. Wang, H. Ren et al., "Fuzzy-based variable structure control for robotic manipulators," Control Theory \& Applications, vol. 24, no. 4, pp. 643-645, 2007, in Chinese.

[10] G. Feng, "A compensating scheme for robot tracking based on neural networks," Robotics and Autonomous Systems, vol. 15, no. 3, pp. 199-206, 1995.

[11] S. Purwar, I. Kar, and A. Jha, "Adaptive output feedback tracking control of robot manipulators using position measurements only," Expert Systems with Applications, vol. 34, no. 4, pp. 2789-2798, 2008.

[12] B. Xu, "Composite learning control of flexible-link manipulator using NN and DOB," IEEE Transactions on Systems, Man, and Cybernetics: Systems, vol. 27, pp. 1-7, 2017.

[13] B. Xu, Y. Shou, J. Luo, H. Pu, and Z. Shi, "Neural learning control of strict-feedback systems using disturbance observer," IEEE Transactions on Neural Networks and Learning Systems, vol. 30, no. 5, pp. 1296-1307, 2019.

[14] F. Liu, L. Liang, L. Qin et al., "Sliding mode control for space manipulator trajectory tracking considering effects of gravity," Control and Decision, vol. 10, pp. 1856-1860, 2014, in Chinese.

[15] F. G. Rossomando and C. M. Soria, "Adaptive neural sliding mode control in discrete time for a SCARA robot arm," IEEE Latin America Transactions, vol. 14, no. 6, pp. 2556-2564, 2016.

[16] X. Wang, F. Wu, H. T. T. Giang et al., “"'Application of linear active disturbance rejection control in full-bridge DC-DC converters," Control Theory \& Applications, vol. 35, no. 11, pp. 67-74, 2018, in Chinese.

[17] J. Han, "From PID to active disturbance rejection control," IEEE Transactions on Industrial Electronics, vol. 56, no. 3, pp. 900-906, 2009.

[18] Z. Gao, "Scaling and parameterization based controller tuning," American Control Conference, vol. 10, pp. 4989-4996, 2003.

[19] Z. Gao, "Active disturbance rejection control: a paradigm shift in feedback control system design," American Control Conference, vol. 10, 2006.

[20] J. Yao and W. Deng, "Active disturbance rejection adaptive control of hydraulic servo systems," IEEE Transactions on Industrial Electronics, vol. 34, 2017.

[21] J. Yao and W. Deng, "Active disturbance rejection adaptive control of uncertain nonlinear systems: theory and application," Nonlinear Dynamics, vol. 56, 2017.
[22] C.-E. Huang, D. Li, and Y. Xue, "Active disturbance rejection control for the ALSTOM gasifier benchmark problem," Control Engineering Practice, vol. 21, no. 4, pp. 556-564, 2013.

[23] H. Xie, K. Song, and Y. He, "A hybrid disturbance rejection control solution for variable valve timing system of gasoline engines," ISA Transactions, vol. 53, no. 4, pp. 889-898, 2014.

[24] J. Yao, Z. Jiao, and D. Ma, "Extended-state-observer-based output feedback nonlinear robust control of hydraulic systems with backstepping," IEEE Transactions on Industrial Electronics, vol. 61, no. 11, pp. 6285-6293, 2014.

[25] Z.-L. Zhao and B.-Z. Guo, "A nonlinear extended state observer based on fractional power functions," Automatica, vol. 81, pp. 286-296, 2017.

[26] Z.-L. Zhao and B.-Z. Guo, "A novel extended state observer for output tracking of MIMO systems with mismatched uncertainty," IEEE Transactions on Automatic Control, vol. 63, no. 1, pp. 211-218, 2018.

[27] C. Sen, W. Xue, S. Zhong et al., "On comparison of modified ADRCs for nonlinear uncertain systems with time delay," Science in China Series F: Information Sciences.vol. 61, p. 7, 2018.

[28] W. Xue and Y. Huang, "Performance analysis of active disturbance rejection tracking control for a class of uncertain LTI systems," ISA Transactions, vol. 77, 2015.

[29] W. Xue and Y. Huang, "Performance analysis of 2-DOF tracking control for a class of nonlinear uncertain systems with discontinuous disturbances," International Journal of Robust and Nonlinear Control, vol. 28, p. 16, 2017.

[30] Y. Huang and W. Xue, "Active disturbance rejection control: methodology and theoretical analysis," ISA Transactions, vol. 53, no. 4, pp. 963-976, 2014.

[31] S. Macfarlane, "Online smooth trajectory planning for manipulators," M.A.Sc. thesis, Department of Mechanical Engineering University, British Columbia, Vancouver, BC, Canada, 2001.

[32] Q. Zheng, L. Q. Gao, and Z. Gao, "On stability analysis of active disturbance rejection control for nonlinear timevarying plants with unknown dynamics," Conference on Decision and Control, vol. 10, pp. 3501-3506, 2007. 